ACCEPTED For PUblicATION IN THE ASTROPHYSiCAL JOURnAL

Preprint typeset using LTEX style emulateapj v. 08/22/09

\title{
NEAR-INFRARED TRANSIT PHOTOMETRY OF THE EXOPLANET HD 149026B
}

\author{
Joshua A. Carter ${ }^{1}$, Joshua N. Winn ${ }^{1}$, Ronald Gilliland ${ }^{2}$, Matthew J. Holman ${ }^{3}$ \\ Accepted for publication in The Astrophysical Journal
}

\begin{abstract}
The transiting exoplanet HD 149026b is an important case for theories of planet formation and planetary structure, because the planet's relatively small size has been interpreted as evidence for a highly metal-enriched composition. We present observations of 4 transits with the Near Infrared Camera and Multi-Object Spectrometer on the Hubble Space Telescope within a wavelength range of 1.1-2.0 $\mu \mathrm{m}$. Analysis of the light curve gives the most precise estimate yet of the stellar mean density, $\rho_{\star}=0.497_{-0.057}^{+0.042} \mathrm{~g} \mathrm{~cm}^{-3}$. By requiring agreement between the observed stellar properties (including $\rho_{\star}$ ) and stellar evolutionary models, we refine the estimate of the stellar radius: $R_{\star}=1.541_{-0.042}^{+0.046} R_{\odot}$. We also find a deeper transit than has been measured at optical and mid-infrared wavelengths. Taken together, these findings imply a planetary radius of $R_{p}=0.813_{-0.025}^{+0.027} R_{\text {Jup }}$, which is larger than earlier estimates. Models of the planetary interior still require a metal-enriched composition, although the required degree of metal enrichment is reduced. It is also possible that the deeper NICMOS transit is caused by wavelength-dependent absorption by constituents in the planet's atmosphere, although simple model atmospheres do not predict this effect to be strong enough to account for the discrepancy. We use the 4 newly-measured transit times to compute a refined transit ephemeris.

Subject headings: stars: individual (HD 149026) — techniques: photometric — stars: planetary systems — stars: fundamental parameters
\end{abstract}

\section{INTRODUCTION}

Since its discovery by Sato et al. (2005), HD 149026b has been one of the most closely scrutinized planets outside the Solar system. It is a close-in gas giant, orbiting a G star with a period of only $2.5 \mathrm{~d}$. Observations of transits (Sato et al. 2005, Charbonneau et al. 2006, Winn et al. 2008b, Nutzman et al. 2008), in combination with observations of radial-velocity variations of the parent star (Sato et al. 2005), have shown that the planet has approximately Saturn's mass but is considerably denser, despite the intense irradiation from the parent star that should inflate the planet and lower its density. There is consensus among theorists that the reason for the "shrunken radius" is a highly metal-enriched composition, although the total metal mass, its distribution within the planet, and the reason for the enrichment are debated [Sato et al. (2005), Fortney et al. (2006), Ikoma et al. (2006), Broeg \& Wuchterl (2007), Burrows et al. (2007)]. The total metal mass, for example, ranges from $60 M_{\oplus}$ to $114 M_{\oplus}$ among the possible models. The latter estimate would represent $80 \%$ of the total mass of the planet.

The planet's outer atmosphere is also of interest, given the possibly unusual composition and the strong heating from the parent star. Models by Fortney et al. (2005) indicated the possibility of a very hot stratosphere as a result of gaseous TiO and VO opacity. By using the Spitzer Space Telescope to observe a planetary occultation, Harrington et al. (2007) found the planet's $8 \mu \mathrm{m}$ brightness temperature to be much larger than the temperature that one would expect based on thermal equilibrium with the incident stellar radiation. This may be the result of the predicted $\mathrm{TiO}$ and $\mathrm{VO}$ heating, although the details of whether and where these absorbers actually condense in the atmosphere are not yet understood (Fortney et al. 2005, Harrington et al. 2007).

Fundamental to all these discussions are the measurements of the mass and radius of HD 149026b. These measurements are limited by the uncertainties in the stellar mass and radius. One way to improve the situation is to observe transits with greater photometric precision than has been possible before. As shown by Seager \& Mallen-Ornelas (2003), with a good light curve and Kepler's third law, one may determine the stellar mean density. If the mean density is known precisely enough, it is a key constraint that can be combined with the other stellar observables (parallax, apparent magnitude, effective temperature, metallicity, etc.) and stellar-evolutionary models to determine the stellar mass and radius. This technique has been put into practice for many other systems [see, e.g., Sozzetti et al. (2007), Holman et al. (2007), Torres et al. (2008)] but never to advantage for HD 149026b because of the limited precision of prior determinations of $\rho_{\star}$ (Winn et al. 2008b, Nutzman et al. 2008). Observers must cope with the small transit depth of 2.5 mmag (smaller than any other transiting exoplanet by a factor of two) and the paucity of suitable comparison stars within the field of view of most telescopes.

In this paper we present observations of transits of HD 149026b with the Near Infrared Camera and Multi-Object Spectrometer [NICMOS, Thompson (1992)] on board the Hubble Space Telescope (HST). We chose this instrument because a high precision in relative photometry is possible even without using comparison stars (Gilliland 2006, Swain et al. 2008) and because the reduced stellar limb-darkening at near-infrared wavelengths is advantageous for the light-curve analysis (Carter et al. 2008, Pál et al. 2008). We have organized this paper as follows. In $\S 2$ we describe the observations and data reductions leading to the final photometric time series. In $\S 3$ we describe our photometric model and the results of the NICMOS light-curve analysis. In

\footnotetext{
Electronic address: carterja@mit.edu

${ }^{1}$ Department of Physics, and Kavli Institute for Astrophysics and Space Research, Massachusetts Institute of Technology, Cambridge, MA 02139

${ }^{2}$ Space Telescope Science Institute, 3700 San Martin Drive, Baltimore, MD 21218

${ }^{3}$ Harvard-Smithsonian Center for Astrophysics, 60 Garden St., Cambridge, MA 02139
} 
$\S 4$, we describe how the light-curve results were incorporated into stellar-evolutionary models to determine the parameters of the HD 149026 system. In $\S 5$, the light analysis is repeated using not only the NICMOS data but also the most precise light curves that have been obtained at optical and mid-infrared wavelengths. In $\S 6$, all the available transit times are analyzed to produce a refined transit ephemeris and to search for possible period variations that could be indicative of additional bodies in the HD 149026 system (Holman \& Murray 2005, Agol et al. 2005, Ford \& Holman 2007). Finally, in $\S 7$, we discuss the possible implications of our observations and analysis.

\section{OBSERVATIONS AND REDUCTIONS}

We observed HD 149026 on four occasions ("visits" in HST parlance) when transits were predicted to occur, on 2007 Dec 22 , 2007 Dec 24, 2008 Feb 08, and 2008 Mar 20. Each visit consisted of five orbits spanning a transit. Between each pair of orbits is an observing gap of approximately 45 minutes, when HST's vision is blocked by the Earth. The visits were scheduled in such a manner that the combined data set provides complete phase coverage of the transit, including redundant coverage of the critical ingress and egress phases. In particular, visits 1 and 3 covered the ingress phase, and visit 2 covered both ingress and egress phases. Visit 4 captured the beginning of egress.

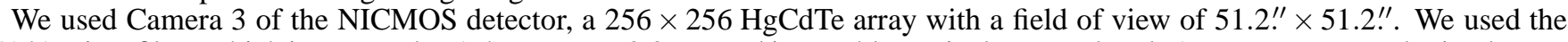
G141 grism filter, which is centered at $1.4 \mu \mathrm{m}$, spans $0.8 \mu \mathrm{m}$ and is roughly equivalent to $H$ band. An exposure was obtained every $13 \mathrm{~s}$. The camera was operated in "MULTIACCUM" mode, wherein five nondestructive readouts are recorded during a single exposure, and the first readout is subtracted from the final readout. After accounting for overheads, the effective integration time was $4 \mathrm{~s}$ per exposure. We deliberately defocused the instrument to give a full-width at half-maximum (FWHM) of approximately 5 pixels in the cross-dispersion direction. This was done for two reasons: firstly, when focused, camera 3 undersamples the point-spread-function (PSF) of point sources; and secondly, the detector pixels exhibit intra-pixel sensitivity variations as large as $30 \%$. Defocusing the images causes the PSF to be well-sampled and averages over the intra-pixel sensitivity variations.

Approximately 220 exposures were collected during each HST orbit. Experience with HST has shown that photometric stability is relatively poor during the first orbit of a given visit. Our observations were scheduled under the assumption that the first orbit from each visit would not be utilized, and indeed we ended up omitting the first-orbit data from our analysis. At the start of each visit, we obtained a single non-dispersed image using a narrow filter centered at $1.66 \mu \mathrm{m}$ in order to establish the pixel position corresponding to zero dispersion. We then adopted the relation from the HST Data Handbook for NICMOS ${ }^{4}$,

$$
\lambda(\Delta x)=-0.007992 \Delta x+1.401
$$

where $\lambda(\Delta x)$ is the wavelength (measured in $\mu \mathrm{m}$ ), and $\Delta x$ is the $x$ coordinate (measured in pixels) relative to the center of the undispersed image.

For completeness, we performed the steps of flat-fielding, background subtraction, and pixel flagging, as described below; however, it is noteworthy that these steps in the data reduction made very little difference in the aperture photometry or in the final results. Flat-field correction for grism images is not straightforward and is not done as part of the standard NICMOS pipeline reductions, because the appropriate flat field depends both upon wavelength and upon the position of the source in the non-dispersed image. To accomplish the flat-field correction, we obtained seven flat fields, each using a narrow bandpass within the G141 bandpass, and fitted the data for each pixel with a quadratic function of wavelength $C[\lambda ; x, y]$. We then applied $C[\lambda(\Delta x) ; x, y]$ as a multiplicative correction to each of our science images, using the wavelength-coordinate relation $\lambda(\Delta x)$ that was determined from the single non-dispersed image. The background level was estimated in each image based on the counts in a relatively clean region of the detector (away from the spectral trace) and subtracted from the entire image. To identify bad pixels, all images from a given orbit were used to create a time series of counts specific to each pixel. Pixels showing an anomalously large variance were flagged. The list of flagged pixels was appended to the list of hot or cold pixels that were identified in the standard NICMOS pipeline reductions, and the values of all of those bad pixels were replaced by interpolated values of the neighboring good pixels.

Aperture photometry was performed on the first-order spectrum, using a simple sum of the counts within a rectangular box centered on the spectral trace. The box had a width of 20 pixels in the cross-dispersion direction (the $y$ direction), which was four times the FWHM of the PSF. The box had a length of 120 pixels in the dispersion direction (the $x$ direction), which was long enough the capture the entire first-order spectrum.

At this stage the data had been reduced to a single number per image: the total number of counts in the aperture (the "flux"). We examined the resulting time series. As expected, the data collected during the first orbits of each visit showed flux variations that were both larger in amplitude and different in their time-dependence than the variations observed in subsequent orbits. The first-orbit data were excluded from subsequent analysis. In addition, we excluded the 10 exposures near the beginning and end of each orbit sequence, because they showed strong flux variations that are probably due to "Earth shine." After these exclusions, there remained 800, 820,800, and 792 good data points in visits $1,2,3$, and 4, respectively.

\section{NICMOS LIGHT-CURVE ANALYSIS}

Fig. 1 shows the time series of the aperture-summed flux, after dividing by the mean flux. In each panel, the zero point of the $x$-axis is the expected mid-transit time. The flux decrement during the transit is identifiable, but this decrement is superimposed on at least two other sources of variability: orbit-to-orbit discontinuities, and smooth intra-orbital variability showing a consistent pattern among all orbits of a given visit. The intra-orbital variability has been seen by all other investigators attempting precise HST photometry of single bright stars, since the pioneering work by Brown et al. (2001), and the flux discontinuities have been seen by other investigators using NICMOS (see, e.g., Swain et al. 2008). The origins of these systematic effects have not been

\footnotetext{
${ }^{4}$ http://www.stsci.edu/hst/nicmos/documents/handbooks/DataHandbookv7/
} 
established. The orbit-to-orbit consistency of the smooth variations suggests a phenomenon that is a function of the phase of the telescope's orbit around the Earth, such as thermal cycling or scattered light. The discontinuities between orbits suggest a non-repeating event associated with the re-aquisition of the target star after each Earth occultation, such as pointing changes or positional shifts of the grism filter.

Ideally, the underlying physical processes giving rise to these systematic effects could be ascertained, and this understanding would lead to either the recognition of an improved method for deriving the photometric signal or a physical model that could be used to correct the aperture-summed flux. Given that we do not yet have such knowledge, what can be done? The intra-orbital variations are very well-described by a smooth function of the HST orbital phase; following other investigators we used a smooth function with several adjustable parameters as an ad hoc model for this variation. The parameters of this model are fairly well constrained by the out-of-transit data, for which all variations are assumed to be systematic effects.

The inter-orbital discontinuities are more problematic. To investigate the systematic effects, we examined the spectral trace on each image. Specifically we computed the flux-weighted mean $y$ position as a function of $x$, giving a curve $y(x)$ representing the centroid of the spectral trace (the "footprint" of the spectrum on the detector). We also estimated the orientation of the spectral trace relative to the detector edges, by performing a linear fit to the previously calculated function $y(x)$. figure 2 shows the results. Within a single orbit, the position and orientation of the spectral trace are relatively constant, as compared to the larger movements that are observed between orbits. The largest variations of the spectral trace (its position and width) seem to coincide with the largest discontinuities in the flux time series.

Given the correlations that are observed between the properties of the spectral trace and the aperture-summed flux, the approach taken by Swain et al. (2008) and other investigators is to "decorrelate" the flux against a number of measured parameters ("state variables") such as the mean $y$ position, cross-dispersion width, orientation angle, and so forth. One way to achieve this decorrelation is to fit linear functions of the state variables to the out-of-transit data, for which all time variations are expected to be due to the systematic effects. Then the best-fitting parameters are used to correct all of the data. Alternatively, one could fit for the linear functions of the state variables simultaneously with the parameters describing the transit light curve.

We attempted both of these procedures and found that while they do reduce the amplitude of the systematic effects, they still leave highly significant systematic variations. We also find this procedure to be undesirable because it is not clear which parameters to include in the fit; because the fitted parameters are highly correlated (the state variables do not vary independently); and because we have no justification for the assumption of a linear function for any of these parameters, without an understanding of the underlying physical effect. An example of a possibly relevant physical effect that would not necessarily be described by a linear function is intra-pixel sensitivity variation, which could lead to a function that is periodic in the pixel coordinates of the spectral trace.

We attempted to fit numerous physically-motivated models (based on the premise of intra-pixel sensitivity variations, among others), and did not find any such model that provided a good fit to the data while also having only a few, nondegenerate adjustable parameters. Ultimately we gave up on attempting to correct the intra-orbital discontinuities based on a priori information. Instead we included in our model an adjustable multiplicative factor specific to each orbit. This might seem devastating to the goal of analyzing the transit light curve, but this is not so. It means that we cannot make use of the relative flux between orbits to derive the transit depth; but as we will show, the truly precious information is in the duration of ingress or egress, which is much less vulnerable to the problem of flux discontinuities. In addition, four of the orbits spanned a full ingress or egress. Data from those four orbits does provide useful information about the transit depth, because the discontinuities appear between orbits and not within orbits.

All together, our model for the flux variation due to systematic effects is

$$
f_{\text {sys }}(t)=f_{o}^{v}\left\{1+c_{0}^{v} \phi(t)+c_{1}^{v}[\phi(t)]^{2}+c_{2}^{v}[\phi(t)]^{3}\right\}
$$

where the $v$ index specifies the visit number (1-4), the $o$ index specifies the orbit number (1-4) within each visit after omitting the first orbit, the 16 numbers $f_{o}^{v}$ are the multiplicative factors describing the inter-orbital discontinuities, $\phi(t)$ is the $H S T$ orbital phase at time $t$, and the 12 numbers $c_{i}^{v}$ (3 per visit) are constants specifying a polynomial function of $\phi$ that describes the intra-orbital variation. The HST orbital phase was defined as

$$
\phi(t) \equiv \frac{(t-\langle t\rangle) \bmod P_{H S T}}{P_{H S T}},
$$

where $P_{H S T}=1.5975$ hours is the orbital period of the HST around the Earth and $\langle t\rangle$ is the midpoint of each orbit's observations. The choice of a polynomial, as opposed to some other smoothly varying function, was arbitrary. We also tried using sinusoidal functions with an angular frequency of $2 \pi / P_{H S T}$, with no significant differences in any of the results described below.

For the transit model, we used the analytic formulas of Mandel \& Agol (2002). Our parameters were the planet-to-star radius ratio $\left(R_{p} / R_{\star}\right)$, the cosine of the orbital inclination $(\cos i)$, the semimajor axis in units of the stellar radius $\left(a / R_{\star}\right)$, the mid-transit time $t_{c}^{v}$, and the two coefficients $u_{1}$ and $u_{2}$ of a quadratic limb-darkening law,

$$
\frac{I_{\mu}}{I_{1}}=1-u_{1}(1-\mu)-u_{2}(1-\mu)^{2}
$$

where $\mu$ is the cosine of the angle between the observer and the normal to the stellar surface and $I_{\mu}$ is the specific intensity as a function of $\mu$. We allowed $u_{1}$ and $u_{2}$ to vary freely, subject to the conditions $u_{1}+u_{2}<1, u_{1}+u_{2}>0$, and $u_{1}>0$. These conditions require the brightness profile to be everywhere positive and monotonically decreasing from limb to center. In practice, the fitting 

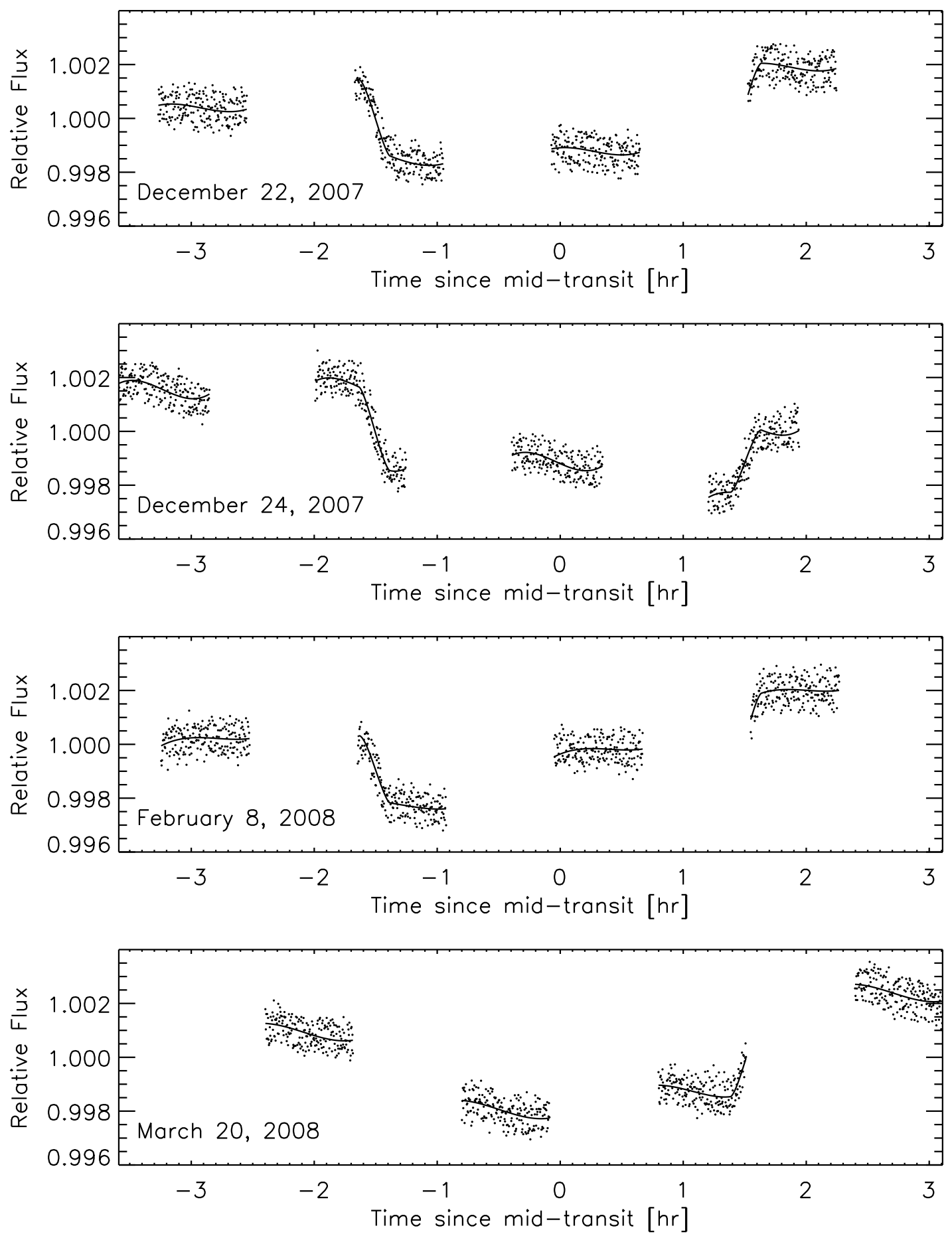

FIG. 1.- NICMOS photometry (1.1-2.0 $\mu \mathrm{m})$ of HD 149026b of 4 transits, with interruptions due to Earth occultations. Plotted are the results of simple aperture photometry. The observed variations are a combination of the transit signal and systematic effects (intra-orbital variations and inter-orbital discontinuities). The solid curve is the best-fitting model that accounts for both the transit signal and systematic effects.

parameters were actually

$$
\begin{aligned}
& u_{1}^{\prime}=u_{1} \cos 40^{\circ}-u_{2} \sin 40^{\circ} \\
& u_{2}^{\prime}=u_{1} \sin 40^{\circ}+u_{2} \cos 40^{\circ}
\end{aligned}
$$

because $u_{1}^{\prime}$ and $u_{2}^{\prime}$ are weakly correlated, unlike $u_{1}$ and $u_{2}$ (Pál 2008). In computing the transit light curve we assumed the orbit to be circular, consistent with the findings of Sato et al. (2005) and Madhusudhan \& Winn (2008). We held the orbital period fixed at the value $P=2.87588$ days based on the results of Winn et al. (2008b). Here the period is used only to relate the measured transit durations and $a / R_{\star}$. The fractional error in $P$ is approximately $10^{4}$ times smaller than the fractional error in $a / R_{\star}$ and is 


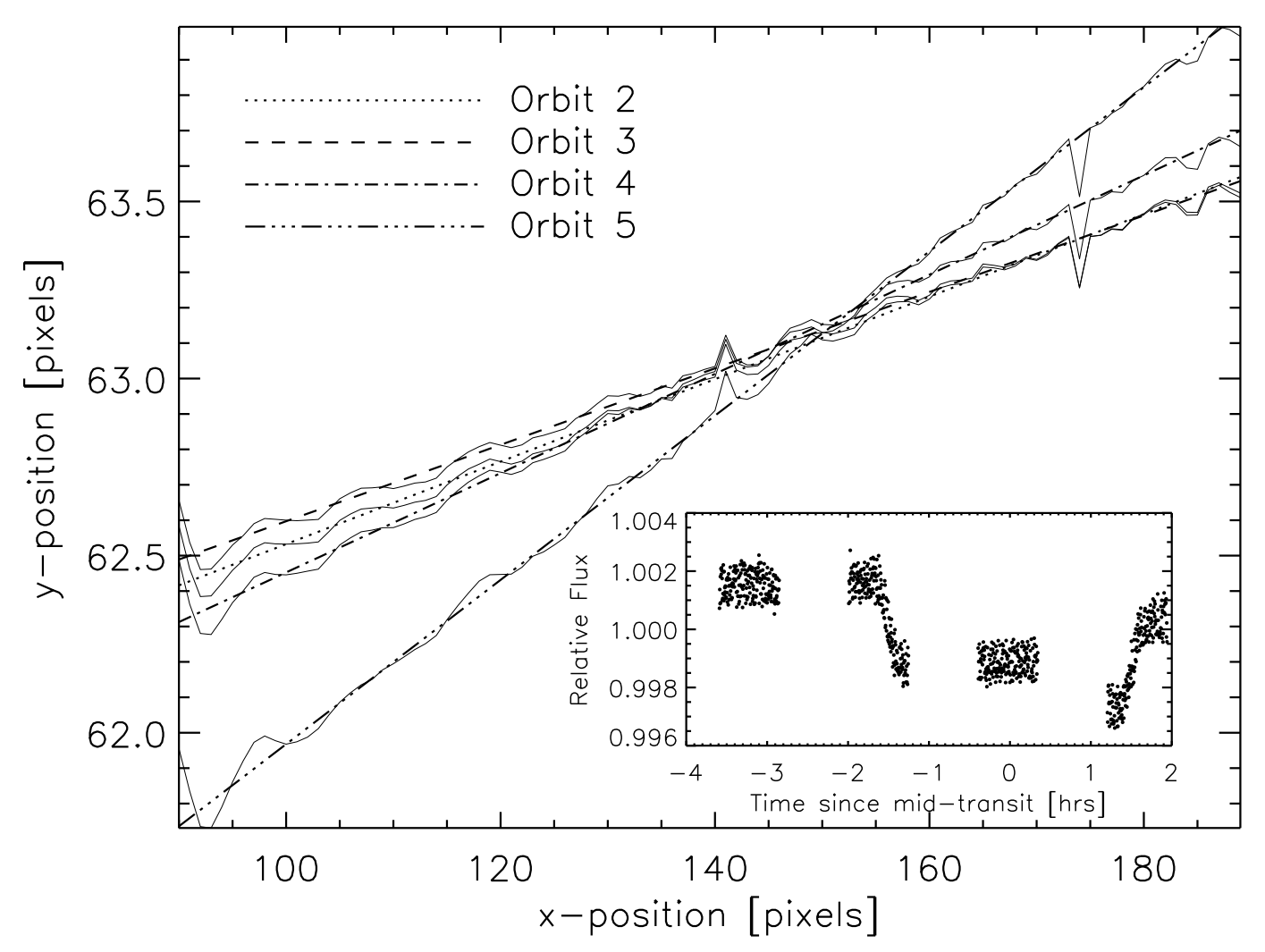

FIG. 2.- Illustration of inter-orbital variations of the spectral trace. The solid curves are the flux-weighted mean $y$ position of the first-order spectrum as a function of $x$. Overplotted are linear fits to $y(x)$. The inset figure shows the measured light curve after dividing out the intra-orbital variations correlated with HST orbital phase. The largest rotation of the spectral trace (at the fifth orbit) coincides with the largest discontinuity in the flux time series.

safely ignored (Carter et al. 2008).

Our complete model of the photometric time series was the product of the transit model and $f_{\text {sys }}(t)$ (Eq. 2). We fitted simultaneously for the parameter set $R_{p} / R_{\star}, \cos i, a / R_{\star}, u_{1}^{\prime}, u_{2}^{\prime},\left\{t_{c}^{v}\right\},\left\{f_{o}^{v}\right\}$, and $\left.\left\{c_{i}^{v}\right\}\right)$. The polynomial describing intra-orbital variations was specific to each visit, and the flux discontinuities were specific to each orbit, but the transit parameters were required to be consistent across all orbits and visits. We performed a least-squares fit to the unbinned data using a box-constrained LevenbergMarquardt algorithm (Levenberg 1944, Marquardt 1963, Lourakis 2004) utilizing the Jacobian calculation of Pál (2008). Box constraints were needed to enforce the restrictions on the limb darkening parameters $u_{1}$ and $u_{2}$. The goodness-of-fit statistic was

$$
\chi^{2}=\sum_{v=1}^{4} \sum_{i=1}^{N_{v}}\left(\frac{f_{\mathrm{obs}}^{v}\left(t_{i}\right)-f_{\mathrm{calc}}^{v}\left(t_{i}\right)}{\sigma_{v}}\right)^{2}
$$

where $f_{\text {mod }}^{v}(i)$ the calculated flux at the time of the $i^{\text {th }}$ data point during visit $v, f_{o b s}^{v}(i)$ is the $i^{\text {th }}$ flux measurement during visit $v, N_{v}$ is the number of data points in visit $v$, and $\sigma_{v}$ was assumed to be a constant at this step. The solid curve in Fig. 1 shows the best-fitting model. The root-mean-square (rms) residual between the data and the best-fitting model is 440 parts per million $(\mathrm{ppm})$. This is approximately 2.2 times the expected noise level calculated within the NICMOS calibration pipeline (which is dominated by photon noise). Fig. 3 shows histograms of the residuals for all the data and for each visit individually. The residuals are not Gaussian; the flattened peak in the histograms indicates a nonzero kurtosis ${ }^{5}$.

Evidently the noise is not photon-limited, and is not Gaussian, but at least it does not appear to be strongly correlated in time. We assessed the degree of temporal correlations ("red noise") in two ways. First, we binned the residuals in time by a factor $N$ ranging from 1 to 100 , and calculated the standard deviation $\sigma_{N}$ of the binned residuals. The results are shown in Fig. 4. They follow closely the expectation of independent random numbers, $\sigma_{N}=\sigma_{1} N^{-1 / 2}[M /(M-1)]^{1 / 2}$, where $M$ is the number of bins.

\footnotetext{
${ }^{5}$ One may wonder about the effect of the apparent non-Gaussianity of the noise, shown in Fig. 3. To investigate this issue we used an Edgeworth expansion to create a new $\chi^{2}$-like statistic that accounts for the skewness and kurtosis of the residuals (see, e.g., Amendola et al. 1996). Using this different fitting statistic, we found that the best-fit parameter values were unchanged. This was not surprising because bias is expected to arise from skewness (as opposed to kurtosis) and the skewness of the residuals is very small. However, the confidence intervals are affected by the kurtosis. We found that accounting for kurtosis leads to error bars that are smaller than the error bars quoted here, but only by a modest amount $(\lesssim 20 \%)$. For simplicity, the results quoted in this paper are based on the standard $\chi^{2}$ statistic given in Eq. (7).
} 
Second, we calculated the Allan (1964) variance $\sigma_{A}^{2}(l)$ of the residuals, defined as

$$
\sigma_{A}^{2}(l)=\frac{1}{2(N+1-2 l)} \sum_{i=0}^{N-2 l}\left(\frac{1}{l} \sum_{j=0}^{l-1} r_{i+j}-r_{i+j+l}\right)^{2}
$$

where $r_{k}$ denotes the residual of the $k$ th data point, $N$ is the number of data points, and $l$ is the lag. The Allan variance is commonly used in the time metrology literature to assess $1 / f$ noise. For independent residuals, one expects $\sigma_{A}^{2}(l) \approx \sigma_{A}^{2}(0) / l$. The results for our data, also shown in Fig. 4, satisfy this expectation. There is no readily identifiable time-correlated component in the time series of residuals.

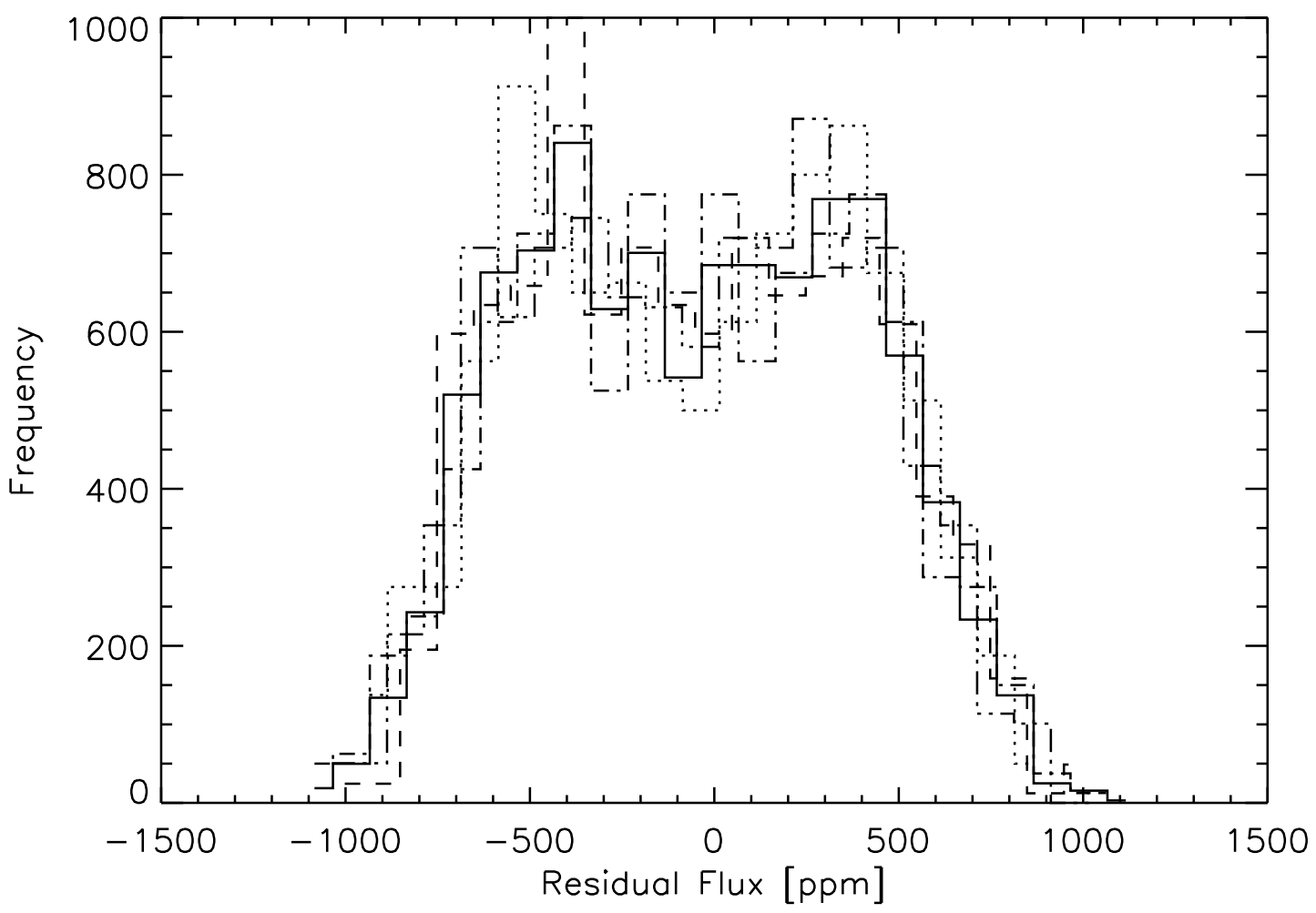

FIG. 3. - Histograms of the residuals between the data and the best-fitting model. The solid line is the histogram based on all the data. The other lines are for data specific to visit 1 (dotted), visit 2 (dashed), visit 3 (dash-dot), and visit 4 (dash-dot-dot-dot).

Plotted in Fig. 5 is the measured flux after dividing by the optimized function $f_{\text {sys }}(t)$. This represents our best effort to correct for the systematic effects. In Fig. 6, we show the results of combining the data from all the visits (after correcting for systematic effects) into a single transit light curve. In this composite light curve, the median time between samples is 7.2 s. Finally, in Fig. 7 we show a time-binned version of the composite light curve to allow a visual comparison with the best previously-measured light curves, at optical and mid-infrared wavelengths.

To determine the "allowed range" of each parameter-or, more precisely, the a posteriori joint probability distribution of all the parameter values-we employed the Markov chain Monte Carlo (MCMC) technique (see, e.g., Winn et al. 2007; Burke et al. 2007). We produced 8 chains of length $7.4 \times 10^{6}$ using a Gibbs sampler and a Metropolis-Hastings jump condition such that each parameter had an effective chain length of roughly $2 \times 10^{5}$. This was accomplished by adjusting the scale of the jump-function distribution such that the probability of accepting a jump is roughly uniform and equal to approximately $40 \%$ across all parameters. To establish initial estimates of parameter uncertainties, a preliminary Monte Carlo bootstrap analysis was performed, based on the Levenberg-Marquardt least squares minimization; then, the MCMC initial conditions were drawn from normal distributions with widths equal to five times these initial error estimates. The first $25 \%$ of each chain was trimmed, and then all the chains were concatenated. Each parameter had a Gelman \& Rubin (1992) $R$ statistic smaller than 1.01, a sign of good convergence of the posterior parameter distributions. For each parameter, the values at each link of the chain were sorted. To describe the results, we report the median (50\%) value, along with the interval between the $15.85 \%$ and $84.15 \%$ levels (the $68.3 \%$ confidence interval). The results are given in Column 2 of Table (1).

\subsection{Results from NICMOS photometric analysis}



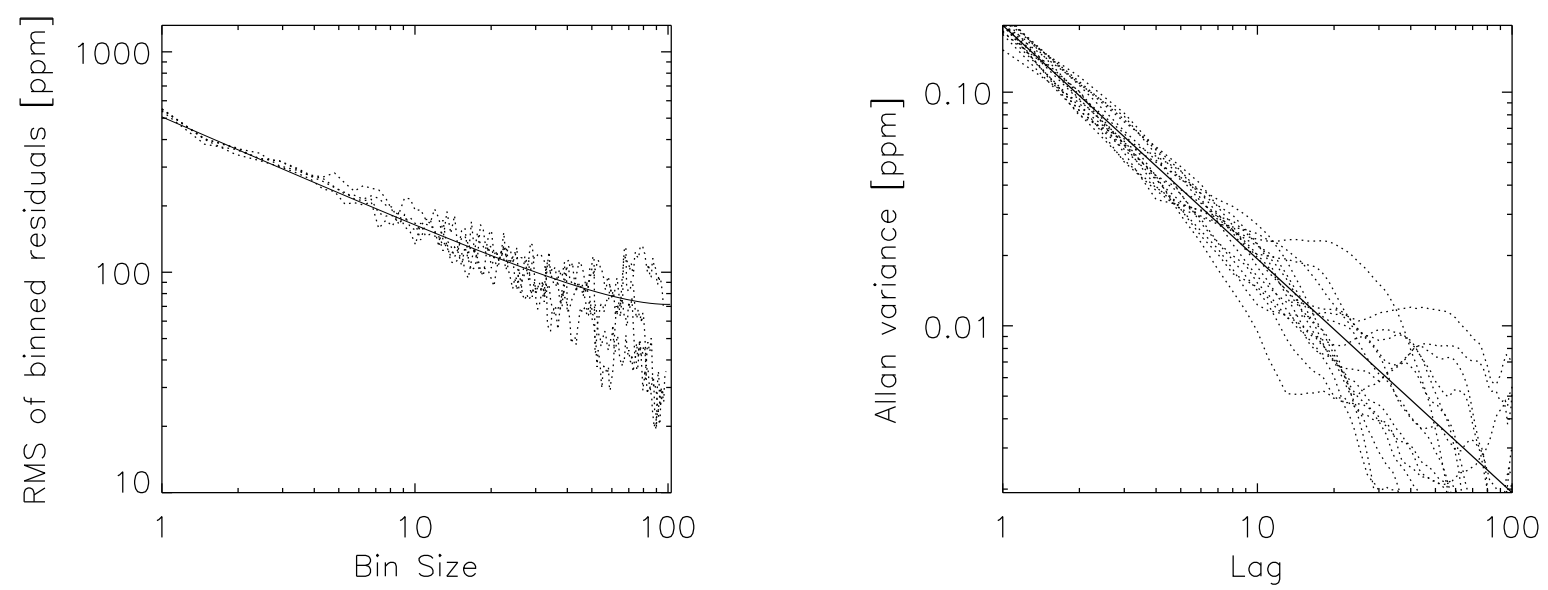

FIG. 4.- Assessment of correlated noise. Left panel: The rms of time-binned residuals, as a function of bin size, for each of the 4 visits. Right panel: The Allan variance of the residuals, as a function of lag, for each of the 16 orbits. The dotted lines are the results of the calculations based on the data, and the solid lines show the expected trend if the noise were uncorrelated.

For the orbital inclination, we find $i=84.55_{-0.81}^{+0.35} \mathrm{deg}$. This is in agreement with the independent estimate of $i=85.4_{-0.8}^{+0.9} \mathrm{deg}$ by Nutzman et al. (2008), using the $8 \mu \mathrm{m}$ channel of the Infrared Array Camera (IRAC) aboard the Spitzer Space Telescope. For the normalized orbital distance, we find $a / R_{\star}=6.01_{-0.23}^{+0.17}$. This is also in agreement with the results of Nutzman et al. (2008), who found $a / R_{\star}=6.20_{-0.63}^{+0.28}$. The new result is more precise, which (as we will show in $\S 4$ ) leads to tighter constraints on the stellar mass and radius. This is important because the uncertainties in the stellar properties have been the limiting factors in the analysis of this system. Based on the preceding results, we find the impact parameter (defined as $b=a \cos i / R_{\star}$ ) to be $0.571_{-0.038}^{+0.044}$. This is the tightest such constraint that has been achieved for HD 149026b. The earliest measurements of the impact parameter were consistent with zero (Sato et al. 2005, Charbonneau et al. 2006, Winn et al. 2008b), a situation leading to strong degeneracies among the transit parameters (Carter et al. 2008). More recently, Nutzman et al. (2008) found $b=0.62_{-0.24}^{+0.08}$, consistent with the new and more precise result. The increased precision in $a / R_{\star}$ and $b$ is a direct consequence of the improved precision with which the ingress (and egress) duration is known (Carter et al. 2008). In this sense, the greatest value of the NICMOS data is in the good coverage of the ingress and egress phases.

The enhanced precision of the NICMOS data relative to previous data sets does not lead to a correspondingly enhanced precision in the planet-to-star radius ratio. This is because we allowed the time series from each orbit to have its own adjustable flux multiplier. Consequently, all of the information about $R_{p} / R_{\star}$ comes only from those orbits that span an entire ingress or egress event. However, the value of $R_{p} / R_{\star}$ that we derive is at least comparable in precision to previous determinations. We find $R_{p} / R_{\star}=0.05416_{-0.00070}^{+0.00091}$. Interestingly this is larger by $2 \sigma$ than the values derived previously, which were based on optical and mid-infrared data. Winn et al. (2008b) found $R_{p} / R_{\star}=0.0491_{-0.0005}^{+0.0018}$ based on Stromgren $(b+y) / 2$ photometry, and Nutzman et al. (2008) found $R_{p} / R_{\star}=0.05158 \pm 0.00077$ based on $8 \mu$ m photometry.

Since we do not understand all of our noise sources with a physically-grounded model, we cannot rule out the possibility that the discrepancy between our result and the previous results is due to a faulty model of the systematic effects. The culprit would probably need to be the polynomial function of orbital phase. We do find that our result is unaffected if we replace the polynomial function of $\phi$ with trigonometric functions, as mentioned previously; and we also find that our results are unchanged if we use a linear limb darkening law or fix the quadratic limb darkening parameters to those tabulated by Claret (2000). These tests do not prove that our results are valid but they do suggest that our analysis procedure is robust to changes in the functional form of the model. However, to the extent that the intra-orbital variations are not strictly repeatable within a given visit, our model would produce biased results. Fig. 8 shows the data after dividing by the optimized values of $f_{o}^{v}$ (the orbit-specific flux multipliers) and dividing by the optimized transit model. The purpose of the divisions is to isolate the intra-orbital systematic effects, which do appear consistent among the orbits within a given visit.

Another possibility for the discrepancy in the transit depth between our near-infrared result and the previous optical and midinfrared results is differential absorption due to constituents in the outer, optically-thin portion of the planet's atmosphere. This interpretation is the basis of the "transmission spectroscopy" technique for identifying constituents of exoplanetary atmospheres pioneered by Charbonneau et al. (2002). We consider this possibility at some length in sections 5 and 7.

We find that the center-to-limb variation is less pronounced than was expected based on the tabulated limb darkening coefficients of Claret (2000). Fig. 9 shows the confidence contours in the $u_{1}, u_{2}$ parameter space. The open square corresponds to the tabulated values for $H$ band (for a star with $T_{\text {eff }}=6250 K, \log g_{\star}=4.5,[\mathrm{Fe} / \mathrm{H}]=0.3$, matching the properties of HD 149026). The tabulated values are excluded with $>95 \%$ confidence.

Two quantities intrinsic to the star and planet that may be calculated directly in terms of observables are the surface gravity of the planet, and the mean density of the star. The surface gravity of the planet is calculated as (Southworth et al. 2007)

$$
g_{p}=\frac{2 \pi}{P} \frac{K}{\left(R_{p} / a\right)^{2} \sin i},
$$



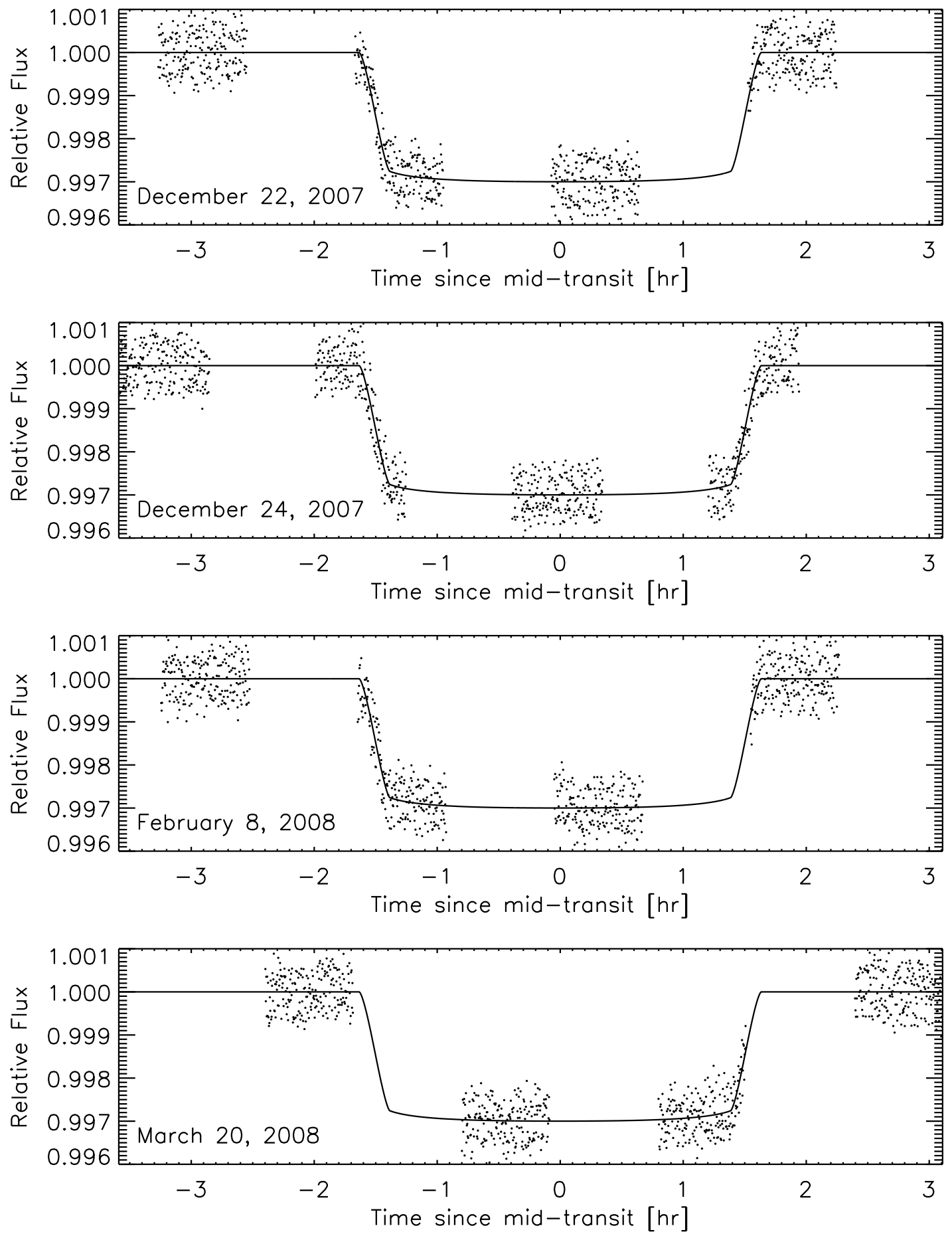

FIG. 5.- NICMOS photometry $(1.1-2.0 \mu \mathrm{m})$ of 4 transits of HD 149026b, after correcting for systematic effects. In each panel, the solid line shows the best-fitting model.

where $K$ is the semiamplitude of the stellar radial-velocity variation (43.3 $\pm 1.2 \mathrm{~m} \mathrm{~s}^{-1}$; Sato et al. 2005), and $R_{p} / a$ and $\sin i$ are derived from our MCMC analysis. We find $\log g_{p}=3.132_{-0.035}^{+0.029}$ where $g_{p}$ is in cgs units. The mean stellar density $\rho_{\star}$ is calculated as (Seager \& Mallen-Ornelas 2003, Sozzetti et al. 2007)

$$
\rho_{\star}=\frac{3 \pi}{G P^{2}}\left(\frac{a}{R_{\star}}\right)^{3}-\rho_{p}\left(\frac{R_{p}}{R_{\star}}\right)^{3}
$$

where $\rho_{p}$ is the mean density of the exoplanet. We may neglect the correction term involving the planetary density as $\rho_{\star} \sim \rho_{p}$, $\left(R_{p} / R_{\star}\right)^{3} \sim 1 \times 10^{-4}$ and the fractional uncertainty in $a / R_{\star}$ is larger than the fractional uncertainty in $R_{p} / R_{\star}$ by a factor of 2 . 

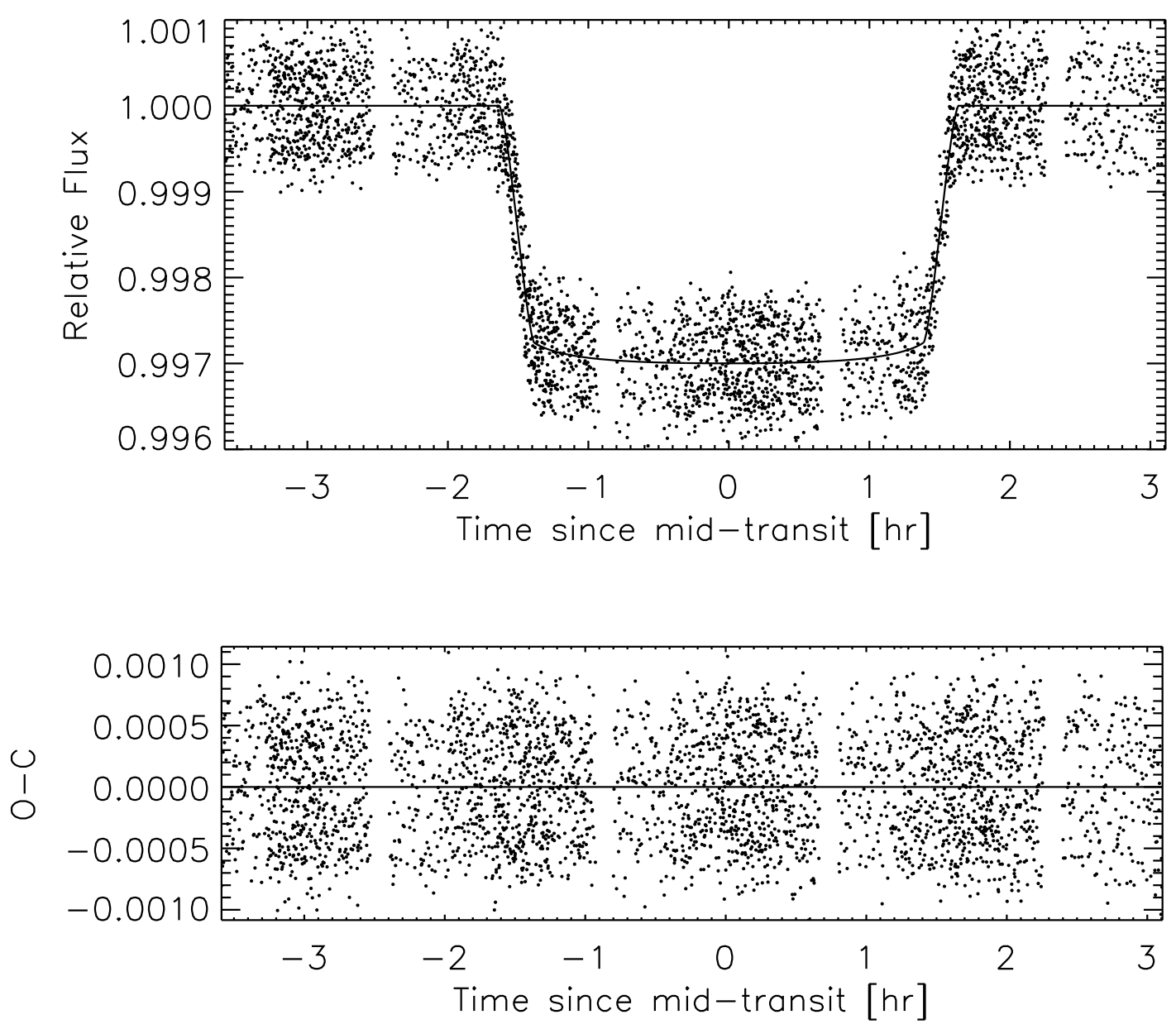

FIG. 6. - NICMOS transit light curve (1.1-2.0 $\mu \mathrm{m})$ of HD 149026b. The data from 4 transits have been superimposed, after correcting for systematic effects. The solid line shows the best-fitting model.

\section{STELLAR PARAMETERS}

The basic inputs to models of the planetary interior are the planetary mass $M_{p}$ and radius $R_{p}$, in units of grams and kilometers, or in units of Jupiter's mass and radius. Transit photometry and Doppler velocimetry alone do not determine these quantities. Additional information about the star must be introduced. Several techniques for estimating the stellar mass $M_{\star}$ and radius $R_{\star}$ were reviewed by Winn et al. (2008b). We chose to estimate $M_{\star}$ and $R_{\star}$ using stellar-evolution models that are constrained by the best available, relevant, observable properties of the star: the mean density $0.497_{-0.057}^{+0.042} \mathrm{~g} \mathrm{~cm}^{-3}$ determined from our light-curve analysis, the absolute magnitude $M_{V}=3.65 \pm 0.12$ derived from the Hipparcos parallax and apparent magnitude $[\pi=12.59 \pm 0.70$ mas, $V=8.15 \pm 0.02$; van Leeuwen (2007)], effective temperature $\left[T_{\text {eff }}=6160 \pm 50 \mathrm{~K}\right.$, a weighted mean of the results from Sato et al. (2005) and Masana et al. (2006)], and metallicity [0.36 \pm 0.08 , from Sato et al. (2005) with a more conservative error bar]. We chose not to use the spectroscopically-determined stellar surface gravity $\left[\log g_{\star}=4.26 \pm 0.07\right.$; Sato et al. (2005)] because the photometrically-determined value of $\rho_{\star}$ provides an effectively tighter constraint, and because spectroscopically-determined surface gravities have been found to be susceptible to systematic error (see, e.g., Winn et al. 2008a, Johns-Krull et al. (2008)).

Following the procedure of Torres, Winn, \& Holman (2008), we employed Yonsei-Yale stellar models ${ }^{6}$ (Yi et al. 2001, Demarque et al. 2004). Model isochrones were interpolated in both age and metallicity, for metallicities $[\mathrm{Fe} / \mathrm{H}]$ ranging from 0.28 to 0.43 and for ages ranging from 0.1 to $14 \mathrm{Gyr}$, in steps of $0.1 \mathrm{Gyr}$. Fig. 10 shows several of these theoretical isochrones, along with some of the observational constraints. The upper left panel illustrates the constraint due to the spectroscopically-determined surface gravity, even though we did not actually apply that constraint, as explained above. It is evident that the constraint due to $\rho_{\star}$ is stronger.

The isochrones were interpolated to provide a fine grid in stellar mass (with a step size of $0.005 M_{\odot}$ ). We then assumed that

${ }^{6}$ We chose the $\mathrm{Y}^{2}$ models mainly because of the convenient form in which they are publicly available. Other stellar-evolutionary models are available, and other investigators have examined the sensitivity of results such as ours to the choice of model. For HD 149026 in particular, Southworth (2008) found that the $\mathrm{Y}^{2}$ models and independent models by Claret (2007) gave results for $M_{\star}$ and $R_{\star}$ that agreed to within $1 \%$. Another set of publicly available models, the Padova models of Girardi et al. (2000), are not computed for the high metallicity observed for HD 149026; but at zero metallicity, at least, both Torres et al. (2008) and Southworth (2008) found that the Padova models give results for the stellar mass and radius that are also within $1 \%$ of the $\mathrm{Y}^{2}$ results. 

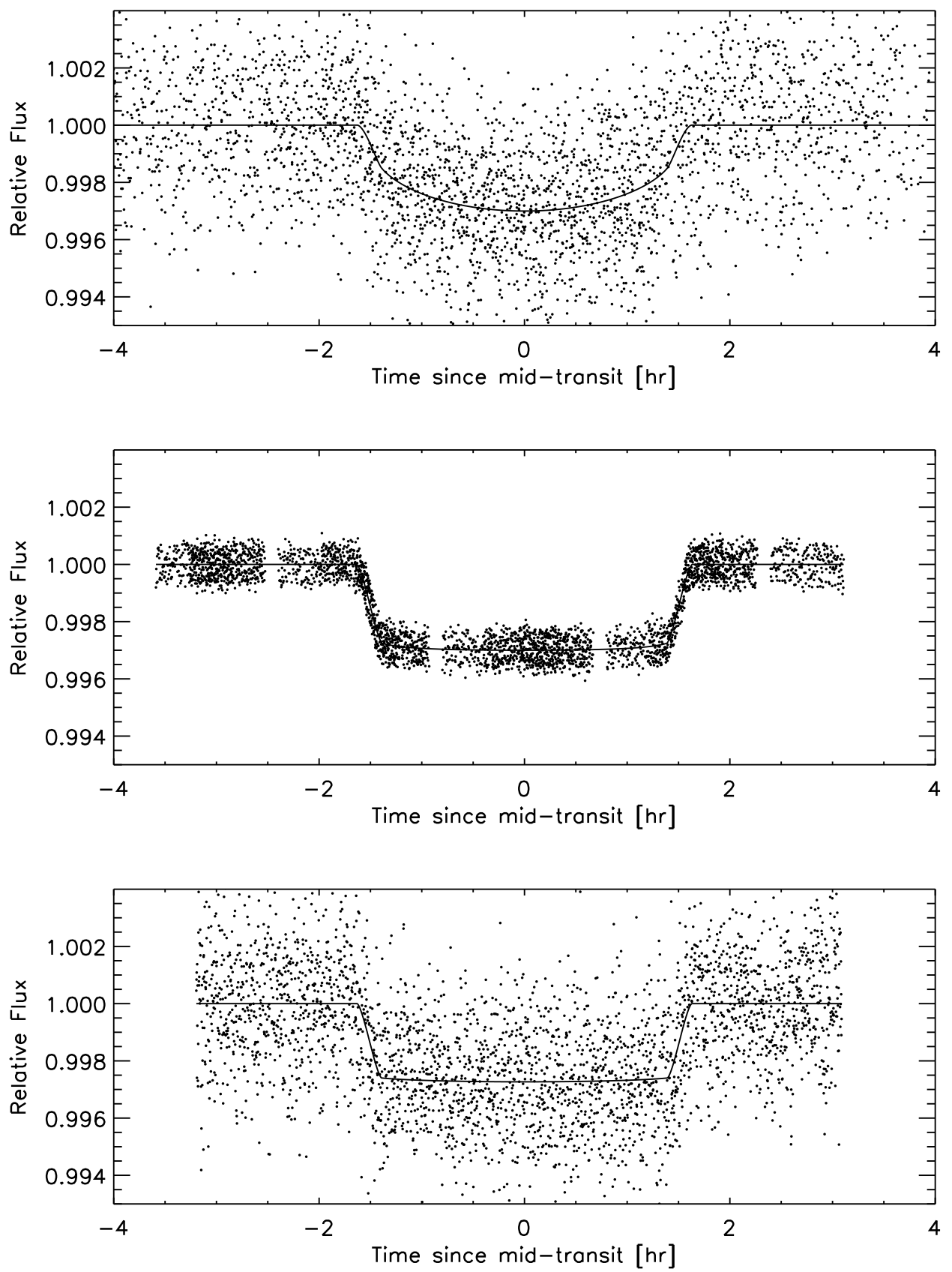

FIG. 7.- Comparison of the best available transit light curves of HD 149026. Top panel: optical [Stromgren $(b+y) / 2]$ photometry from Sato et al. (2005) and Winn et al. (2008b), with a time sampling of $8.6 \mathrm{~s}$ and an rms residual of $2017 \mathrm{ppm}$. Middle panel: near-infrared [1.1-2.0 $\mu \mathrm{m}$ ] photometry from this work, with a time sampling of $7.2 \mathrm{~s}$ and an rms residual of $440 \mathrm{ppm}$. Bottom panel: mid-infrared [8 $\mu \mathrm{m}$ ] photometry from Nutzman et al. (2008), with a time sampling of $7.4 \mathrm{~s}$ and an rms residual of $1854 \mathrm{ppm}$. The solid lines show the best-fitting model, which was calculated as described in $\S 5$.

the likelihood of each point on the interpolated isochrones was proportional to $\exp \left(-\chi_{\star}^{2} / 2\right)$, where

$$
\chi_{*}^{2}=\left(\frac{\Delta[\mathrm{Fe} / \mathrm{H}]}{\sigma_{[\mathrm{Fe} / \mathrm{H}]}}\right)^{2}+\left(\frac{\Delta T_{\text {eff }}}{\sigma_{T_{\text {eff }}}}\right)^{2}+\left(\frac{\Delta M_{V}}{\sigma_{M_{V}}}\right)^{2}+\left(\frac{\Delta \rho_{\star}}{\sigma_{\rho_{\star}}}\right)^{2},
$$

and the $\Delta$ quantities denote the differences between the observed and calculated values. The asymmetry in the error distribution for $\rho_{\star}$ was taken into account. Additionally, the likelihood was taken to be proportional to an Salpeter initial mass function, $\xi(M) \propto M^{-(1+x)}$ with $x=1.35$ (Salpeter 1955). The joint probability function, $P$, was taken to be proportional to the likelihood, viz.,

$$
P\left(R_{\star}, M_{\star}, T_{\text {eff }}, \log g_{\star}, M_{V},[\mathrm{Fe} / \mathrm{H}], \rho_{\star}, \mathrm{Age}\right) \propto \exp \left(-\chi_{*}^{2} / 2\right) .
$$




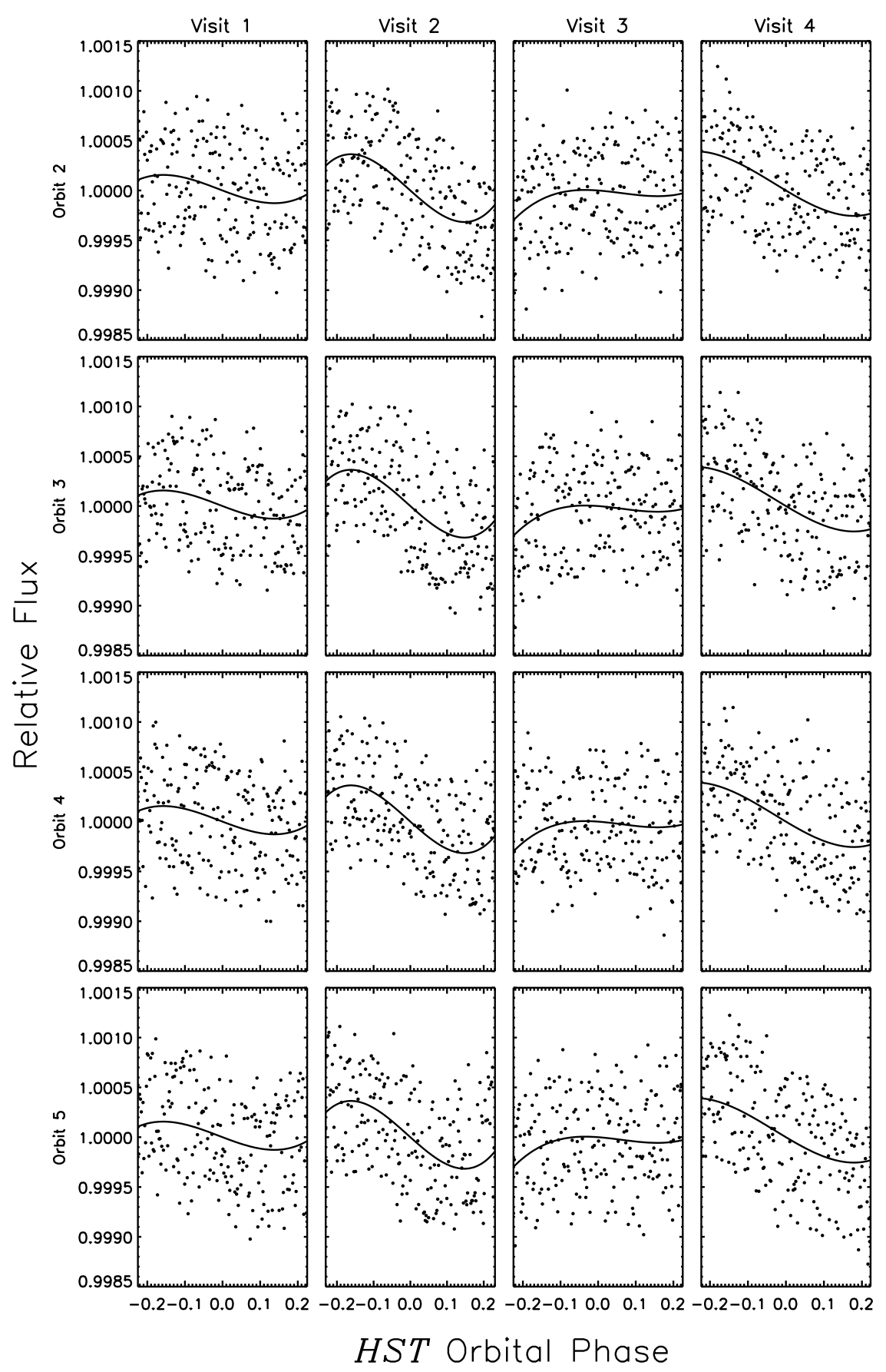

FIG. 8.- Isolation of the intra-orbital variations. The flux time series has been divided by the optimized flux multipliers $f_{o}^{v}$ and by the optimized transit model. The remaining variation appears to present a consistent pattern among all orbits within a given visit, as assumed in our model. The solid line is the optimized model. Each column shows data from all orbits of a given visit. Each row shows orbits arranged from first to last in rows from top to bottom, respectively.

For a given parameter $X_{0}$ from this list, we calculated the cumulative distribution function (CDF) by numerically evaluating

$$
D_{X_{0}}(x)=\int_{-\infty}^{x} d X_{0} \int_{-\infty}^{\infty} d X_{1} \cdots \int_{-\infty}^{\infty} d X_{N} P\left(X_{0}, X_{1}, \cdots, X_{N}\right)
$$

For each parameter, we record the values of $x$ for which the CDF takes the values $15.85 \%, 50 \%$, and $84.15 \%$. The $50 \%$ level (the median) is reported as the "best-fit value" and the interval between the $84.15 \%$ and $15.85 \%$ levels is reported as the $68.3 \%(1 \sigma)$ confidence interval. ${ }^{7}$

\footnotetext{
${ }^{7}$ Although our procedure was inspired by the work of Torres et al. (2008) and is similar in almost all respects, there is one significant difference. The best fit values reported by Torres et al. (2008) were those that minimized $\chi^{2}$ as in our analysis. The difference is that Torres et al. (2008) estimated the $1 \sigma$ errors in the stellar properties based on the total span of the calculated values that gave agreement within $1 \sigma$ with the observables. Effectively, they assumed a uniform
} 


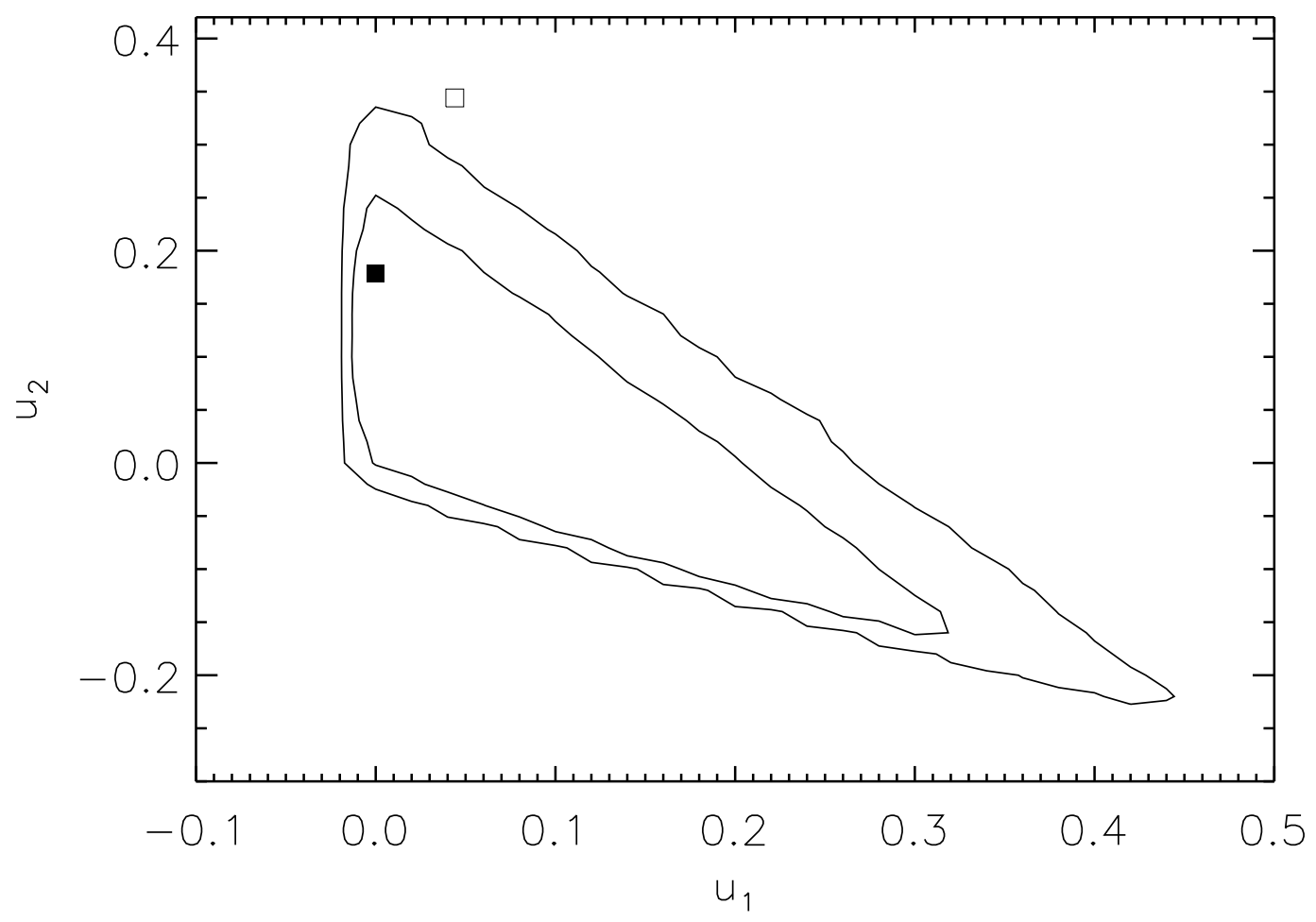

FIG. 9.- Results for the limb-darkening parameters $u_{1}$ and $u_{2}$. The contours are the $68 \%$ and $95 \%$ confidence regions as determined by the MCMC analysis of the photometric time series. The solid square is the minimum- $\chi^{2}$ solution, $u_{1}=0.0, u_{2}=0.1789$. The open square marks the tabulated values of Claret (2000) for $H$ band $\left(u_{1}=0.044, u_{2}=0.344\right)$.

Table (1) reports the best-fit stellar parameters and confidence intervals. We find the stellar radius to be $R_{\star}=1.541_{-0.042}^{+0.046} R_{\odot}$. This is larger than (but in agreement with) the previous estimates of $R_{\star}=1.46 \pm 0.10 R_{\odot}$ by Sato et al. (2005), and $R_{\star}=$ $1.497 \pm 0.069 R_{\odot}$ ) by Nutzman et al. (2008). By combining the derived distribution for $R_{\star}$ with the photometrically-determined distribution for $R_{p} / R_{\star}$, we find the planetary radius to be $R_{p}=0.813_{-0.025}^{+0.027} R_{\mathrm{Jup}}$. This is larger than any previous result. Using optical photometry, Sato et al. (2005) found $0.725 \pm 0.050 R_{\mathrm{Jup}}$, Charbonneau et al. (2006) found $0.726 \pm 0.064 R_{\mathrm{Jup}}$, and Winn et al. (2008b) found $0.71 \pm 0.05 R_{\text {Jup }}$. Using mid-infrared photometry, Nutzman et al. (2008) found $0.755 \pm 0.040 R_{\text {Jup }}$. It is important to note that these determinations were not wholly independent, and therefore should not be combined into a weighted average. They all used many common inputs for the stellar properties, and the analyses of optical photometry all included a common subset of at least 3 light curves.

As mentioned previously, we did not apply any constraint to the models based on the spectroscopically determined value of $\log g_{\star}$. However, given our results for $M_{\star}$ and $R_{\star}$ we computed the implied value of $\log g_{\star}$, finding $\log g_{\star}=4.189_{-0.021}^{+0.020}$. This in agreement with, and is more precise than, the spectroscopically-determined value of $\log g_{\star}=4.26 \pm 0.07$ (Sato et al. 2005).

\section{JOINT ANALYSIS WITH OPTICAL AND MID-INFRARED LIGHT CURVES}

Transit observations of HD 149026b have now been made at optical wavelengths (Sato et al. 2005, Charbonneau et al. 2006, Winn et al. 2008b), near-infrared wavelengths (this work), and mid-infrared wavelengths (Nutzman et al. 2008). In this section we repeat our analysis on all of these data, in order to bring all of these data to bear on the determination of the system parameters, while seeking possible wavelength variations in the planet-to-star radius ratio.

It is reasonable to require consistency across these data in the parameters relating to the orbital configuration of the transit, such as the inclination angle and normalized semi-major axis. However, the inferred planet-to-star radius ratio is a wavelengthdependent quantity, depending on the opacity of the exoplanetary atmosphere and the emergent flux from the planetary nightside (which is expected to be unimportant). With this in mind, we performed a joint analysis of all of the data, requiring consistency in $\cos i$ and $a / R_{\star}$ but allowing $R_{p} / R_{\star}$ to take separate values for each of the three types of data: optical, near-infrared, and mid-infrared.

Specifically, we fitted our NICMOS data, the $8 \mu \mathrm{m}$ IRAC time series of Nutzman et al. (2008), and the 8 light curves obtained in the Strömgren $(b+y) / 2$ band by Sato et al. (2005) and Winn et al. (2008b). Our photometric model for the NICMOS data,

error distribution for each observable, rather than a Gaussian error distribution as we have done. Consequently, our method produces smaller error intervals in the stellar properties. Caution would dictate that larger error intervals are desirable, especially since we are relying on the theoretical isochrones that surely have some unaccounted-for systematic errors. However, using a uniform error distribution for the observables is an arbitrary way to inflate the output errors, and the true error distribution for the observables is probably closer to Gaussian. For these reasons we chose our approach and emphasize the caveat that our results place complete trust in the $\mathrm{Y}^{2}$ isochrones. 

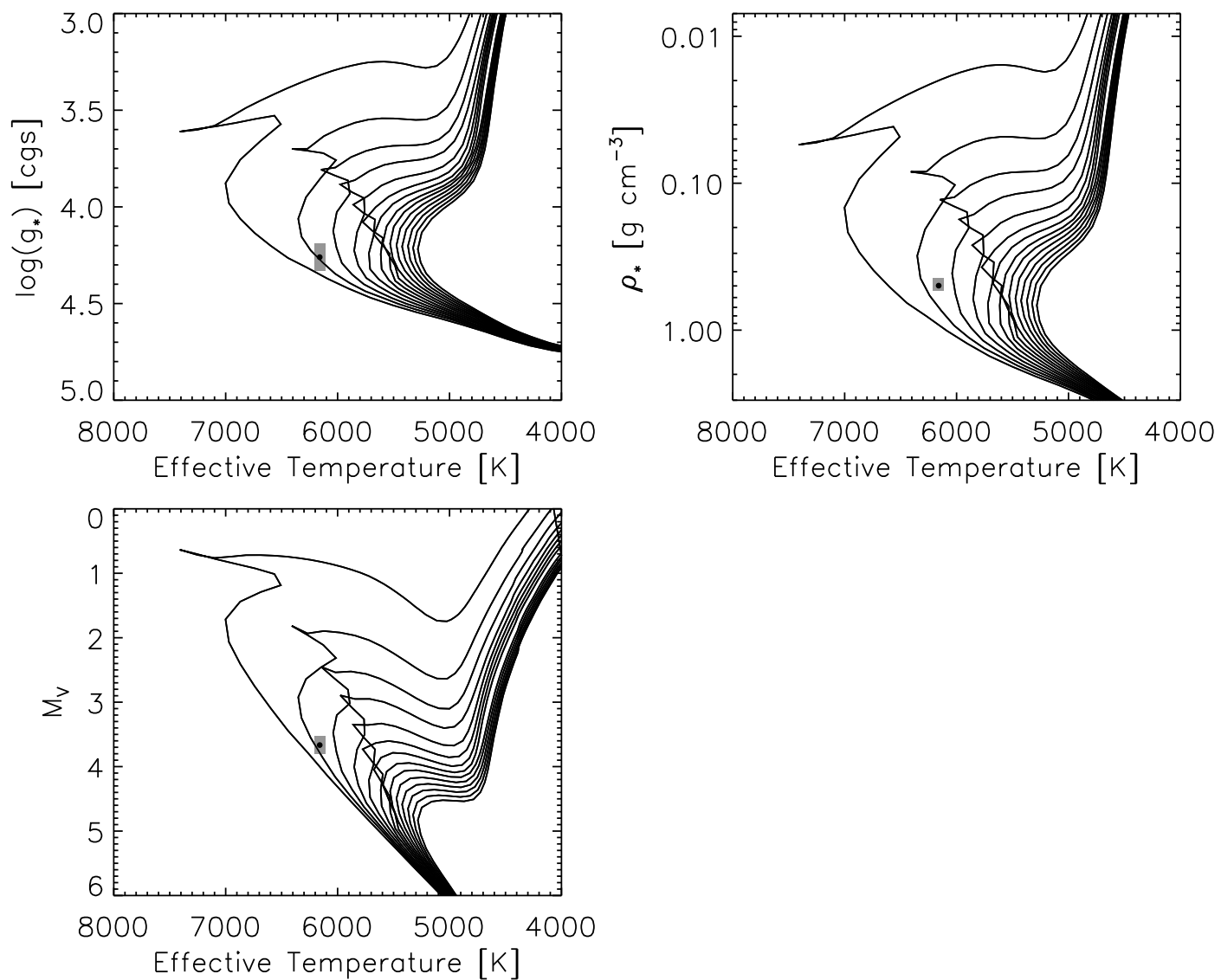

FIG. 10.- Stellar-evolutionary model isochrones, from the Yonsei-Yale series by Yi et al. (2001). The points and shaded boxes represent the observationallydetermined values and $1 \sigma$ errors. Here, surface gravity is determined spectroscopically (Sato et al. 2005), $M_{V}$ is derived from Hipparcos parallax and $V$ magnitudes, and $\rho_{\star}$ is determined photometrically from the transit light curve. Isochrones are shown for ages of 1 to $13 \mathrm{Gyr}$ (from left to right) in steps of $1 \mathrm{Gyr}$ for a fixed stellar metallicity of $[\mathrm{Fe} / \mathrm{H}]=0.36$

including the associated systematic effects, has already been described. For the data sets presented by other authors, we followed those authors' prescriptions to account for systematic errors. For the $(b+y) / 2$ data, we corrected the data by allowing the outof-transit flux to be a linear function of time. For the $8 \mu \mathrm{m}$ data, we modeled the time-variable sensitivity of the detector (the "ramp") as a multiplicative correction, $f_{\text {sys }}=a_{0}+a_{1} \log \left(t-t_{0}\right)+a_{2} \log ^{2}\left(t-t_{0}\right)$, where $t_{0}$ is the time immediately prior to the start of the observation.

We performed an MCMC analysis of this joint data set. The free parameters relating to the transit model were the three values of $R_{p} / R_{\star}$ (corresponding to the ratios measured at approximately $0.5 \mu \mathrm{m}, 1.5 \mu \mathrm{m}$, and $8.0 \mu \mathrm{m}$ ); the geometric parameters $\cos i$ and $a / R_{\star}$; the quadratic limb-darkening coefficients for the NICMOS light curve; the linear limb-darkening coefficients for the optical and infrared light curves (for which the precision of the data do not justify the more accurate quadratic law); and the mid-transit times for the NICMOS data and the IRAC data. ${ }^{8}$ We also fitted for the ramp-correction terms for the IRAC data and the parameters relating to the flux offsets and intra-orbital variations for the NICMOS data. Six chains of length $9 \times 10^{6}$ were created, representing approximately $2 \times 10^{5}$ correlation-lengths per parameter. These were concatenated after removing the first $25 \%$ of each chain. The Gelman-Rubin $R$ statistic was smaller than 1.01 for each parameter. We then repeated the analyses that were described in $\S 3$ and $\S 4$ to determine the stellar, planetary, and orbital parameters, based on this joint analysis. The results are tabulated in Table (1).

The results for the geometric parameters are hardly changed from the NICMOS-only analysis, a reflection of the greater precision of the NICMOS light curve. The planet-to-star radius ratio was found to be larger for the NICMOS data than for the other bandpasses, as was already evident from the comparison of our NICMOS-only analysis to previously published analyses. Fig. 13 shows the variation in $\left(R_{p} / R_{\star}\right)^{2}$ with wavelength. The quantity $\left(R_{p} / R_{\star}\right)^{2}$ is essentially the transit depth, or fractional loss of light during the total phase of the transit, after "removing" the effects of limb darkening. The radius ratios that we derive for the mid-infrared and optical data are in agreement with those reported previously. For the IRAC data we find $R_{p} / R_{\star}=$ $0.05188_{-0.00086}^{+0.00084}$ as compared to the value $0.05158 \pm 0.00077$ reported by Nutzman et al. (2008). For the $(b+y) / 2$ data we find $R_{p} / R_{\star}=0.05070_{-0.00088}^{+0.00058}$ as compared to the value $0.0491_{-0.0005}^{+0.0018}$ found by Winn et al. (2008b). The precision in the optical $R_{p} / R_{\star}$

\footnotetext{
${ }^{8}$ To keep the number of parameters as small as possible, we did not vary the optical mid-transit times or baseline correction parameters at this stage, having found that they are uncorrelated with the other parameters of interest.
} 
has been increased because the NICMOS data pins down all of the other parameters that are correlated with $R_{p} / R_{\star}$.

\section{EPHEMERIS AND TRANSIT TIMING}

The NICMOS-only analysis resulted in the measurement of four distinct mid-transit times, with uncertainties smaller than $45 \mathrm{~s}$. These are given in Table (2). We pooled together all of the independent measurements of mid-transit times from Winn et al. (2008b), Nutzman et al. (2008), and this work, to derive a new transit ephemeris. We fitted the times to a linear function of the integral epoch $E$,

$$
T_{c}(E)=T_{c}(0)+E P
$$

where $P$ is the period and $T_{c}(0)$ is the mid-transit time at some fiducial epoch. The results were $T_{c}(0)=2454456.78761 \pm 0.00014$ HJD and $P=2.8758911 \pm 0.0000025$ days. The linear fit had $\chi^{2}=20.16$ and 14 degrees of freedom. This is a marginally unacceptable fit. The formal probability to find a value of $\chi^{2}$ this large is $15 \%$. Further transit observations are needed to distinguish the possibilities of a genuine period variation, a statistical fluke, and underestimated timing errors. Fig. 11 shows the $\mathrm{O}-\mathrm{C}$ (observed minus calculated) timing diagram.

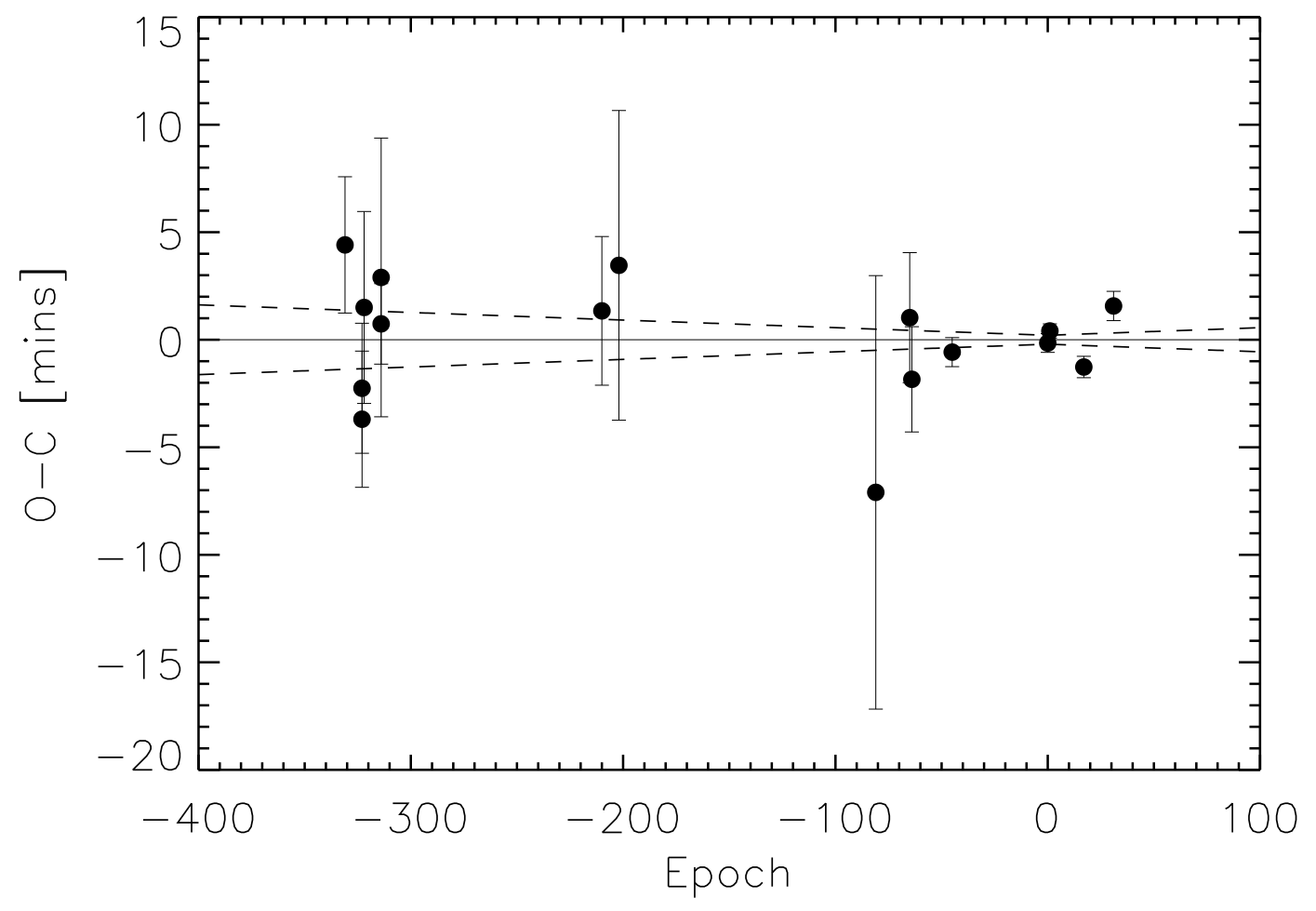

FIG. 11.- Transit-timing variations for HD 149026b. The differences between observed and calculated mid-transit times are plotted. The last 4 points represent the new NICMOS observations. The dashed lines show the $1 \sigma$ range in the calculated times according to the linear ephemeris presented in $\S 6$.

\section{DISCUSSION}

We have presented observations of four transits of HD $149026 \mathrm{~b}$ at near-infrared wavelengths with the HST NICMOS detector. The NICMOS data place the strongest constraints yet on the geometrical system parameters. In particular, the increased precision of the measurement of the normalized semi-major axis $\left(a / R_{\star}\right)$ leads to an improved estimate of the mean stellar density, which was then coupled with stellar evolution models to constrain the stellar mass and radius. Improved knowledge of the stellar mass and radius leads to greater precision in the planetary mass and radius. We have found a larger stellar radius, and a larger planetto-star radius ratio, than previous estimates. As a result of these two factors, we have also found the planetary radius to be larger than previously thought. The planet has "grown" by about $7 \%$.

Despite this increase, our results are still consistent with the contention that HD 149026b is highly enriched in heavy elements. It is still smaller than expected for a hydrogen-helium planet with the given mass and degree of stellar irradiation (Burrows et al. 2007). For comparison, the tabulated models by Fortney et al. (2007) predict a $1.3 R_{\text {Jup }}$ hydrogen-helium HD $149026 \mathrm{~b}$ at an age of 1 Gyr. A variety of models have been developed to estimate the heavy-element content of HD 149026b (Sato et al. 2005, Fortney et al. 2006, Ikoma et al. 2006, Burrows et al. 2007), most of which suppose that the metals are confined to an inner core of material beneath a hydrogen-helium envelope. Other physical considerations in these models include the equation of 
state for heavy elements at core pressures, atmospheric opacities and the upper boundary condition where energy is delivered from the star. To determine a revised estimate for the heavy-element content, we used the tabulated models provided by Sato et al. (2005) and Fortney et al. (2007), and interpolated the tabulated results as appropriate for the planetary radius, planetary mass, and degree of irradiation that follow from the parameters determined from the NICMOS data. We find a core mass in the range of $45-70 M_{\oplus}$, depending on assumed stellar age and core density. Thus, the interpretation of the planet as highly enriched is unaffected, although the required amount of enrichment is slightly reduced.

It is also interesting that the planet-to-star area ratio, $\left(R_{p} / R_{\star}\right)^{2}$, was found to be $2-3 \sigma$ larger in the NICMOS band $(1.1-2.0 \mu \mathrm{m})$ than in the optical band $(0.45-0.55 \mu \mathrm{m})$ or mid-infrared bands $(6.5-9.5 \mu \mathrm{m})$, while the results for the latter two bands are in agreement. Caution dictates that this discrepancy should not be over-interpreted. It is possible that the discrepancy is at least partly the result of unresolved systematic errors in any of the data sets. We have already noted that the noise in the NICMOS data exceeds the photon noise level by a factor of 2 , and is not well understood.

However, it is also worth considering that this wavelength-dependent variation represents selective absorption by constituents in the outermost layer of the planet's atmosphere. Molecules with strong absorption bands at near-infrared wavelengths would cause the transit to appear deeper at those wavelengths. Strong bands are expected for the common molecules $\mathrm{CO}, \mathrm{H}_{2} \mathrm{O}$, and $\mathrm{CH}_{4}$ (Brown 2001, Hubbard et al. 2001, Seager \& Sasselov 2000). If this were the case, then a detailed analysis of the NICMOS spectrophotometry - breaking it down into smaller wavelength bins, as opposed to summing the entire first-order spectrummight be used to identify some constituents of the planet's atmosphere. In addition, more care would be needed in choosing which radius to use in the comparison with models of the planet's interior. It is beyond the scope of this paper to analyze the wavelength dependence of the transit depth across the NICMOS band, or to compute a realistic atmospheric model to see if the contrast between the optical, near-infrared, and mid-infrared results can be accommodated. We can, however, perform an order-of-magnitude calculation to check on the plausibility of this interpretation.

Let $z(\tau)$ be the height in the planet's atmosphere at which the optical depth is $\tau$ for a path from the star to the observer, as diagrammed in Fig. 12. This height is measured relative to an atmospheric base radius $R_{0}$, where the planet is optically thick at all relevant wavelengths. The height $z(\tau)$ depends, in part, on the wavelength-dependent opacity and the density profile of the atmosphere. We define $R_{p}(\lambda)$ as $R_{0}+z(\tau=1)$ and $\delta \equiv\left(R_{p} / R_{\star}\right)^{2}$. If we assume that $z(1) \ll R_{0}$, then the transit depth is approximately linear in $z(1)$ :

$$
\delta=\frac{\left[R_{0}+z(1)\right]^{2}}{R_{\star}^{2}} \approx\left(\frac{R_{0}}{R_{\star}}\right)^{2}\left[1+\frac{2 z(1)}{R_{0}}\right] .
$$

Next, we consider the difference in $\delta$ as measured in two distinct wavelength bands:

$$
\delta_{1}-\delta_{2} \approx \frac{2 R_{0}}{R_{\star}^{2}}\left[z_{1}(1)-z_{2}(1)\right]=\frac{2 R_{0}}{R_{\star}^{2}} \delta z
$$

where we have defined the height difference $\delta z \equiv z_{1}(1)-z_{2}(1)$. The height difference $\delta z$ reflects differing levels of absorption in the two bands. Solving for $\delta z$ in Eqn. (16) we find

$$
\delta z=\frac{1}{2}\left[\frac{\delta_{1}-\delta_{2}}{\left(R_{0} / R_{\star}\right)^{2}}\right] R_{0}
$$

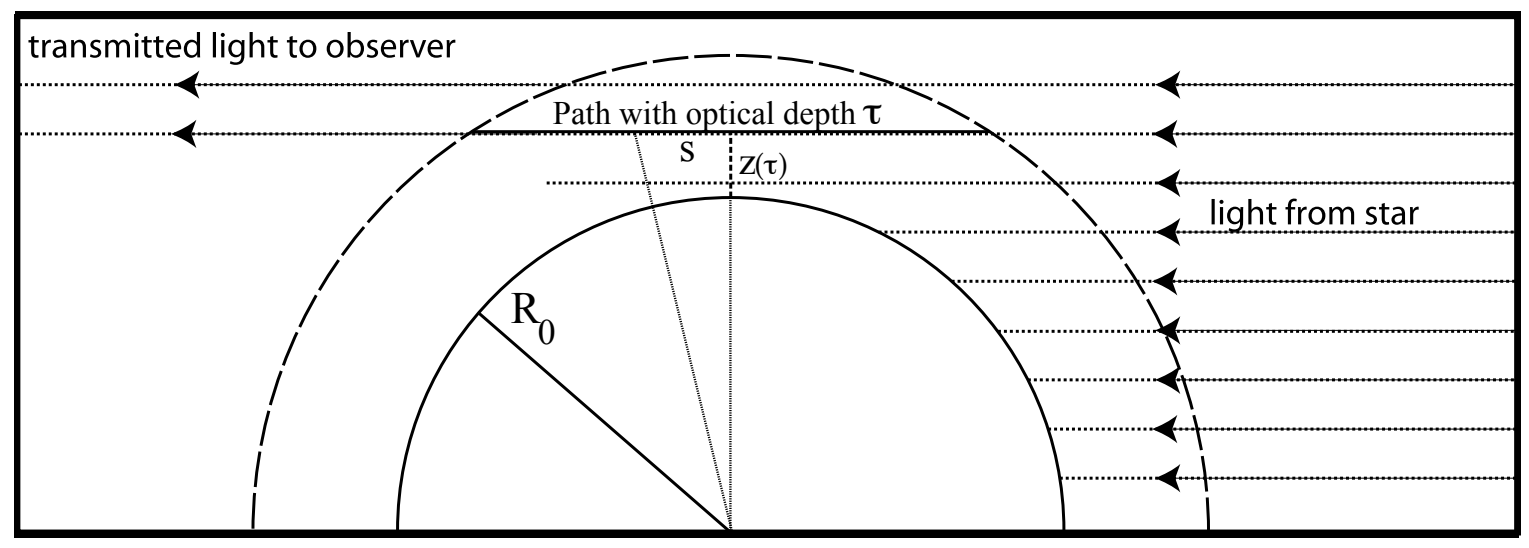

FIG. 12.- Illustration of wavelength-dependent absorption. Shown are some rays that skirt the planetary atmosphere on their way to Earth. At some height $z(\tau)$ above the fiducial radius $R_{0}$, the ray (parameterized by path length $s$ ) has an optical depth of $\tau$ (solid line). Light that follows paths with $z<z(\tau=1$ ) are mainly absorbed. The height $z(\tau=1)$ corresponding to optical depth of unity depends on wavelength, giving rise to a wavelength-dependent transit radius.

For HD 149026b, to evaluate the idea that the larger near-infrared measurement of $\delta$ is due to molecular absorption, we assume that $R_{0}$ is the optically-derived radius $\left(R_{0}=0.757 R_{\mathrm{Jup}}\right)$ because the optical spectrum is expected to show comparatively weak absorption features (Brown 2001, Seager \& Sasselov 2000). Using Eqn. (17), the 0.035\% difference in $\delta$ between near-infrared and mid-infrared wavelengths implies $\delta z \approx 2500 \mathrm{~km}\left(4 \%\right.$ of $\left.R_{0}\right)$. To judge if this is realistic, we are interested in expressing $\delta z$ 
in units of the pressure scale height, for which an order-of-magnitude expression is $H=k T / \mu m_{p} g_{p}$, where $T$ is a representative atmospheric temperature, $\mu$ is the mean molecular weight of the atmosphere, $m_{p}$ is the proton mass and $g_{p}$ is the surface gravity. Using the surface gravity that was determined from our analysis of the optical light curve $\left(g_{p}=1535 \mathrm{~cm} \mathrm{~s}^{-2}\right)$, and assuming a $\mathrm{H}_{2}-\mathrm{He}$ atmosphere with $T=2300 \mathrm{~K}$ [as measured at $8 \mu \mathrm{m}$ by Harrington et al. (2007)], we find $H \approx 530 \mathrm{~km}$, and $N_{H} \approx 5$. If instead we use the planet's predicted temperature at thermal equilibrium with the incident stellar radiation $(T=1700 \mathrm{~K})$, we find $H \approx 400 \mathrm{~km}$ and $N_{H} \approx 6$.

If we assume further that the absorbers have an exponential density profile,

$$
\rho(z)=\rho(0) \exp \left(-\frac{z}{h}\right)
$$

where $h$ is the density scale height, then we may express $z(\tau)$ in terms of the opacity $\sigma$ of the absorbing molecules, as follows. By integrating the optical depth $\tau$ across the optical path at height $z \ll R_{0}$ (as illustrated in Fig. 12), where a change of path length $d s$ results in a change in optical depth $d \tau$ as

$$
d \tau=-s \sigma \rho(0) \frac{\exp \left[-\frac{R_{0}}{h}\left(\sqrt{\left(1+z / R_{0}\right)^{2}+\left(s / R_{0}\right)^{2}}-1\right)\right]}{\sqrt{\left(1+z / R_{0}\right)^{2}+\left(s / R_{0}\right)^{2}}} \frac{d s}{R_{0}},
$$

we find

$$
\frac{z(\tau)}{h} \approx \ln \left(\frac{2 h \sigma \rho(0)}{\tau}\right)-1
$$

In general, $h$ may be different for each atmospheric constituent. If we assume that the components are uniformly mixed throughout the atmosphere, then $h$ is independent of composition, and the difference between two heights $z_{1}(\tau)$ and $z_{2}(\tau)$ at two distinct wavelength bands with different opacities $\sigma_{1}$ and $\sigma_{2}$ can be written independently of the optical depth, as

$$
\delta z=z_{1}-z_{2} \approx h \ln \left(\frac{\sigma_{1}}{\sigma_{2}}\right) .
$$

If we assume further that the temperature scale height is large compared to the pressure scale height, then $h \approx H$ and $N_{H} \approx$ $\ln \left(\sigma_{1} / \sigma_{2}\right)$.

We may now judge the plausibility of this interpretation with reference to the typical opacities and widths of molecular absorption features. For strong molecular bands and atomic lines, the ratio of the in-band opacity to the nearby continuum opacity may be as large as $10^{4}$ (Brown 2001, Seager \& Sasselov 2000), yielding a maximum height difference of $N_{H} \approx 10$ scale heights within the absorption band. The NICMOS band from 1.1-2.0 $\mu$ m includes strong rotation-vibration molecular absorption bands due to $\mathrm{H}_{2} \mathrm{O}, \mathrm{CO}$ and $\mathrm{CH}_{4}$. An example of a very strong absorption band is a water band centered at $1.4 \mu \mathrm{m}$, spanning approximately $10 \%$ of the effective filter width. If we assume that this is the dominant spectral feature in this band, then the result of $N_{H} \approx 5$ across the entire bandpass translates into $N_{H} \approx 50$ within the bandpass of the absorption feature. This is larger than the criterion $N_{H} \approx 10$ mentioned above.

Therefore this interpretation seems to require significantly more opaque or broader-band absorption features than are seen in the models. In one sense the result of the order-of-magntiude calculation is discouraging, as it may make it seem more likely that the discrepancy in depths is due to systematic errors. On the other hand, if the noise were well-understood and the discrepancy could be confidently proclaimed, then it would be the sign of new and interesting atmospheric physics that is not described in the standard models. Some priorities for progress on this issue include an examination of the wavelength-dependence of the transit across the NICMOS band, and the observation of the system with other NICMOS grisms, which are reputed to be more stable than the G141 grism used here.

We thank G. Torres for helpful discussions concerning the determination of stellar parameters. This work was supported by NASA grant HST-GO-11165 from the Space Telescope Science Institute, which is operated by the Association of Universities for Research in Astronomy, Incorporated, under NASA contract NAS5-26555.

\section{REFERENCES}

Agol, E., Steffen, J., Sari, R., \& Clarkson, W. 2005, MNRAS, 359, 567

Amendola, L. 1996, MNRAS, 283, 983

Broeg, C., \& Wuchterl, G. 2007, MNRAS, 376, L62

Brown, T. M. 2001, ApJ, 553, 1006

Burke, C. J., et al. 2007, ApJ, 671, 2115

Burrows, A., Hubeny, I., Budaj, J., \& Hubbard, W. B. 2007, ApJ, 661, 502

Carter, J. A., Yee, J. C., Eastman, J., Gaudi, B. S., \& Winn, J. N. 2008, ApJ, 689,499

Charbonneau, D., et al. 2006, ApJ, 636, 445

Charbonneau, D., Brown, T. M., Burrows, A., \& Laughlin, G. 2007,

Protostars and Planets V, 701

Claret, A. 2000, A\&A, 363, 1081
Claret, A. 2007, A\&A, 467, 1389

Demarque, P., Woo, J.-H., Kim, Y.-C., \& Yi, S. K. 2004, ApJS, 155, 667

Ford, E. B., \& Holman, M. J. 2007, ApJ, 664, L51

Fortney, J. J. 2005, MNRAS, 364, 649

Fortney, J. J., Marley, M. S., \& Barnes, J. W. 2007, ApJ, 659, 1661

Fortney, J. J., Saumon, D., Marley, M. S., Lodders, K., \& Freedman, R. S. 2006, ApJ, 642, 495

Gelman, A., Rubin D. B. 1992, Statistical Science, 7, 457

Gilliland, R. L. 2006, The 2005 HST Calibration Workshop: Hubble After the Transition to Two-Gyro Mode, 162

Girardi, L., Bressan, A., Bertelli, G., \& Chiosi, C. 2000, A\&AS, 141, 371 
Harrington, J., Luszcz, S., Seager, S., Deming, D., \& Richardson, L. J. 2007, Nature, 447, 691

Holman, M. J., \& Murray, N. W. 2005, Science, 307, 1288

Hubbard, W. B., Fortney, J. J., Lunine, J. I., Burrows, A., Sudarsky, D., \& Pinto, P. 2001, ApJ, 560, 413

Ikoma, M., Guillot, T., Genda, H., Tanigawa, T., \& Ida, S. 2006, ApJ, 650, 1150

Johns-Krull, C. M., et al. 2008, ApJ, 677, 657

Levenberg, K., 1944, The Quarterly of Applied Mathematics, 2, 164

Lourakis, M. I. A., 2004,

http://www.ics.forth.gr/ lourakis/levmar

Mandel, K., \& Agol, E. 2002, ApJ, 580, L171

Masana, E., Jordi, C., \& Ribas, I. 2006, VizieR Online Data Catalog, 345, 735

Marquardt, D., 1963, SIAM Journal on Applied Mathematics, 11, 431

Nutzman, P., Charbonneau, D., Winn, J. N., Knutson, H. A., Fortney, J. J.,

Holman, M. J., \& Agol, E. 2008, ArXiv e-prints, 805, arXiv:0805.0777

Pál, A. 2008, MNRAS, 390, 281

Salpeter, E. E. 1955, ApJ, 121, 161
Seager, S., \& Mallén-Ornelas, G. 2003, ApJ, 585, 1038

Seager, S., \& Sasselov, D. D. 2000, ApJ, 537, 916

Sato, B., et al. 2005, ApJ, 633, 465

Southworth, J. 2008, arXiv:0811.3277

Southworth, J., Wheatley, P. J., \& Sams, G. 2007, MNRAS, 379, L11

Sozzetti, A., Torres, G., Charbonneau, D., Latham, D. W., Holman, M. J.,

Winn, J. N., Laird, J. B., \& O’Donovan, F. T. 2007, ApJ, 664, 1190

Swain, M. R., Vasisht, G., \& Tinetti, G. 2008, ArXiv e-prints, 802,

arXiv:0802.1030

Thompson, R. 1992, Space Science Reviews, 61, 69

Torres, G., Winn, J. N., \& Holman, M. J. 2008, ApJ, 677, 1324

van Leeuwen, F. 2007, A\&A, 474, 653

Winn, J. N., et al. 2008, ApJ, 683, 1076

Winn, J. N., Holman, M. J., \& Roussanova, A. 2007, ApJ, 657, 1098

Winn, J. N., Henry, G. W., Torres, G., \& Holman, M. J. 2008, ApJ, 675, 1531

Yi, S., Demarque, P., Kim, Y.-C., Lee, Y.-W., Ree, C. H., Lejeune, T., \& Barnes, S. 2001, ApJS, 136, 417

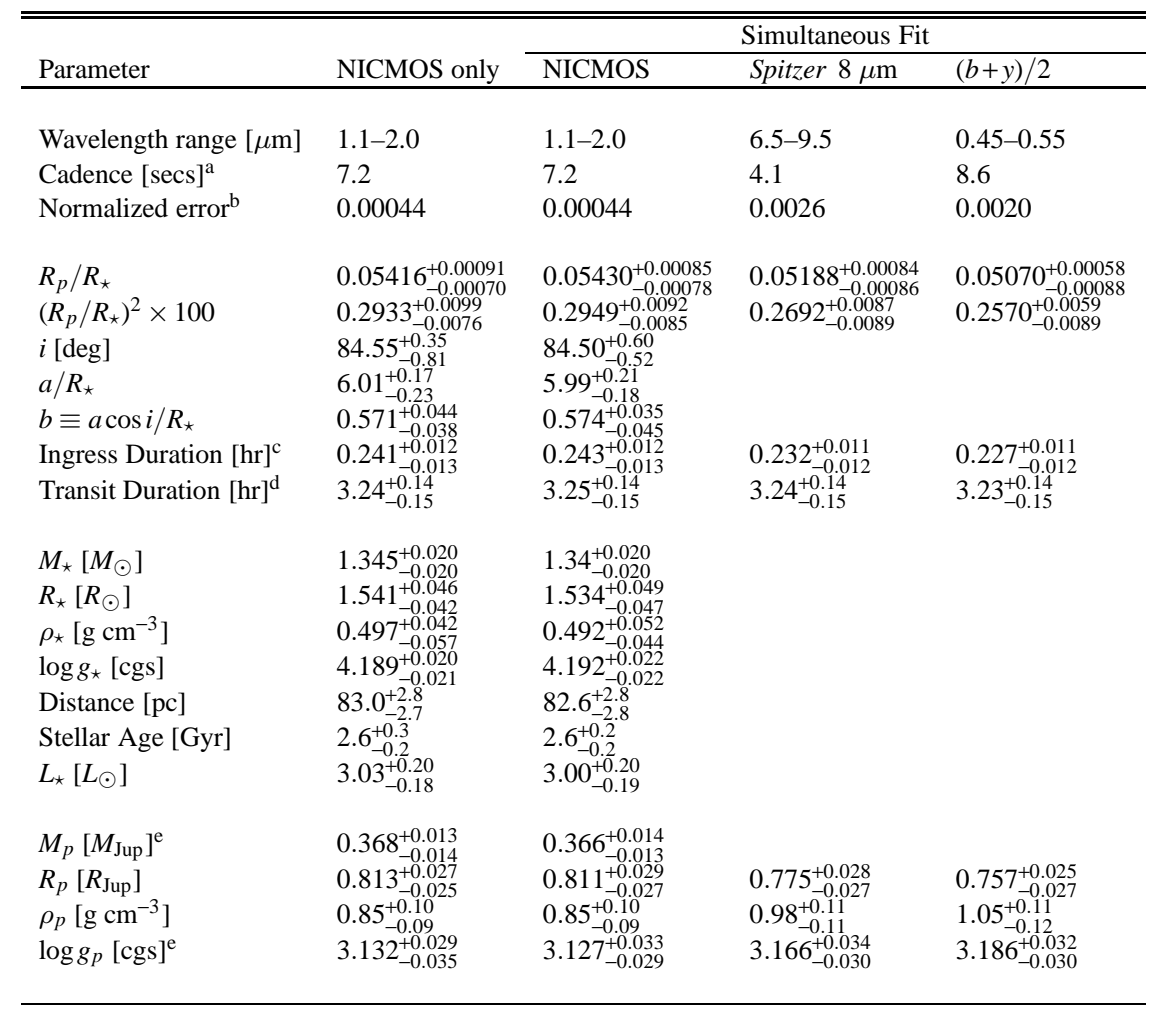

TABLE 1

SYSTEM PARAMETERS OF HD 149026.

${ }^{a}$ Defined as the median time interval between data points in the composite (phase-folded) light curve.

${ }^{\mathrm{b}}$ Defined as $\sigma_{F} / F$ where $\sigma_{F}$ is the rms residual between the data and best-fitting model, and $F$ is the out-of-transit flux.

${ }^{c}$ Defined as the time between first and second contacts, or between third and fourth contacts. (In our model these durations must be equal.)

${ }^{\mathrm{d}}$ Defined as the time between the first and fourth contacts.

${ }^{\mathrm{e}}$ Using $K=43.3 \pm 1.2 \mathrm{~m} \mathrm{~s}^{-1}$, from Sato et al. (2005).

\begin{tabular}{lll}
\hline \hline Epoch & Mid-transit time [HJD] & Error \\
\hline 0 & 2454456.78751 & 0.00030 \\
1 & 2454459.66379 & 0.00023 \\
17 & 2454505.67688 & 0.00034 \\
31 & 2454545.94133 & 0.00047 \\
\hline
\end{tabular}

TABLE 2

Mid-TRANSit TIMES, BASED ON THE NICMOS DATA. 


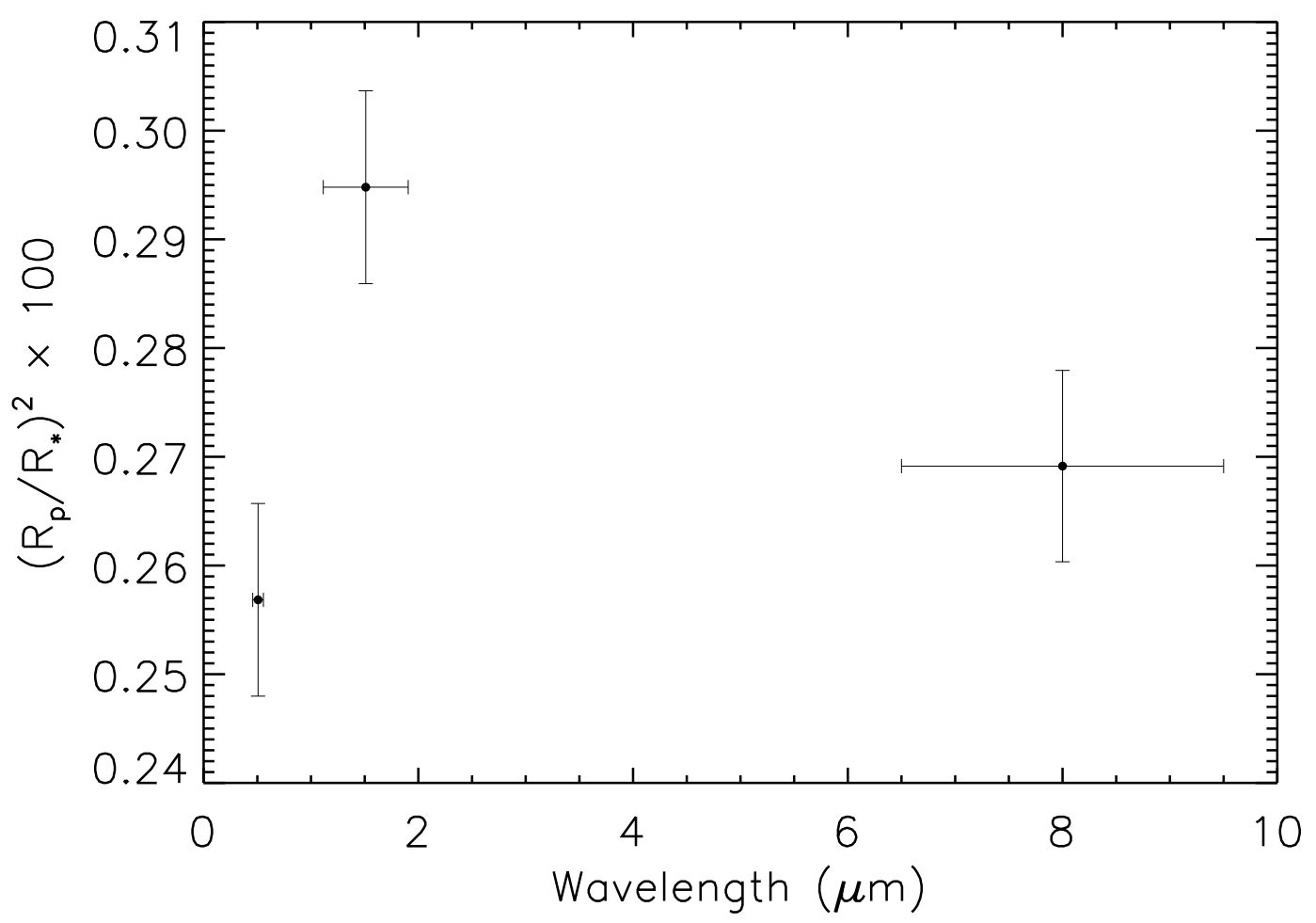

FIG. 13. - The planet-to-star area ratio, $\left(R_{p} / R_{\star}\right)^{2}$, as a function of observing wavelength, based on a joint fit to the NICMOS data, the $(b+y) / 2$ data of Winn et al. (2008), and the IRAC data of Nutzman et al. (2008). The horizontal error bars show the approximate wavelength range of each bandpass. 